تحلال لتصادي قيلمي المحدلت الهاءة النسوقية ومحدلت صاكرت البلملس المصربة

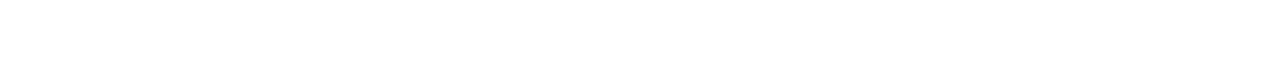

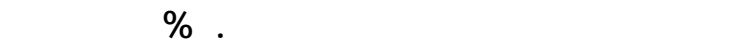

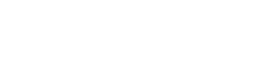

$$
\text { .r. Ir - } 1994
$$

0 - تززلي إلجملل كمية وقيمة صلارك البمالملس المصرية خلال

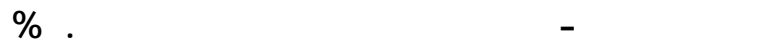

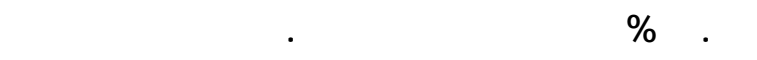

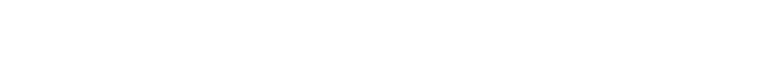

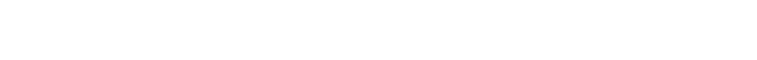

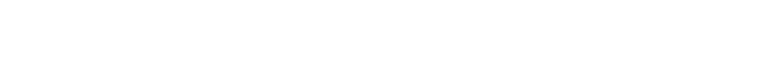

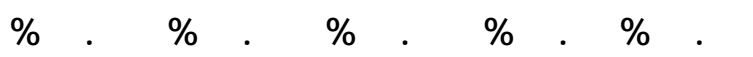
عل الترتيب من إجمالل كمية المالرك المصربة من ون

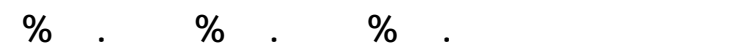

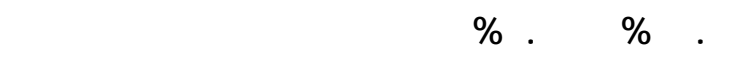

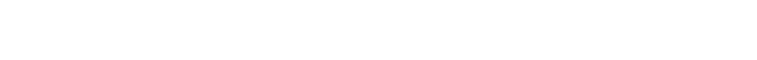

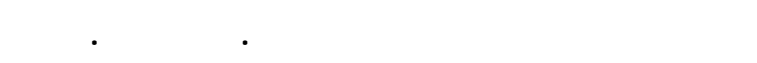

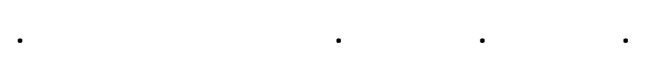

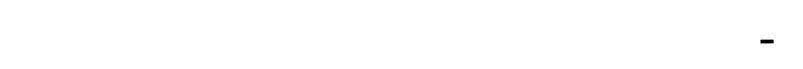

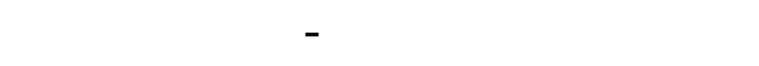
التصير المصري للان وسعر التصير العالي للالن بمتوطا بلغ حوال

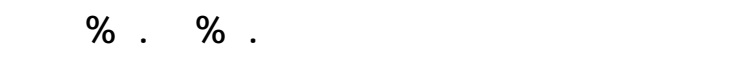
الترتيب عندمستوك معنوية 1 . . . . V - Vبلغ متوسط مؤشر معالل عم الإسقرار لكمية الصع الرات

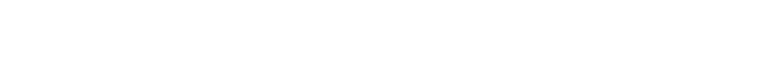

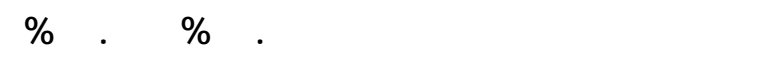

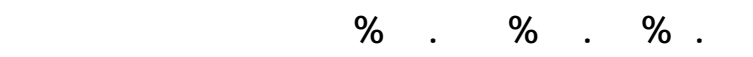

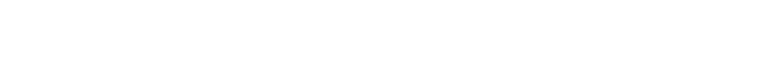

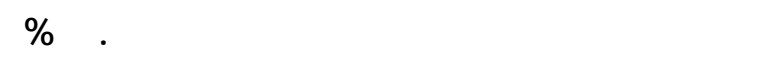

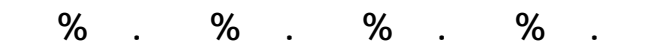

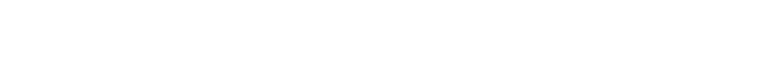

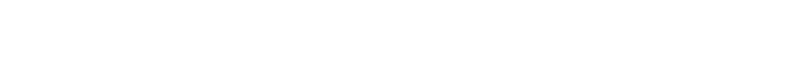

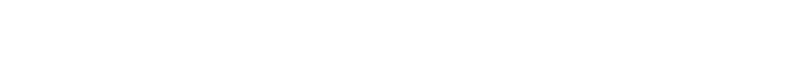

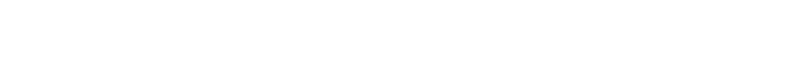

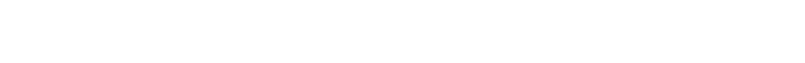

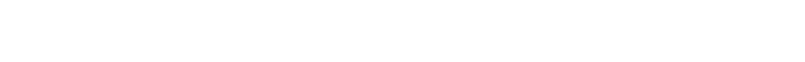

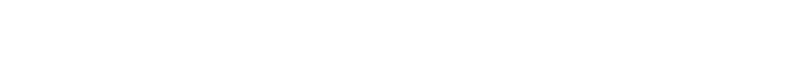

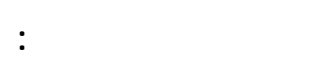

1 - عمم حصوث تغيرلت مالموسة في الهاءة النسوقية لمحصط

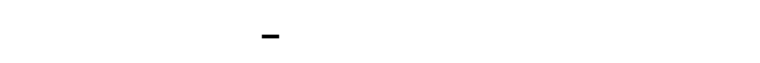

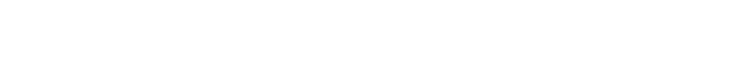

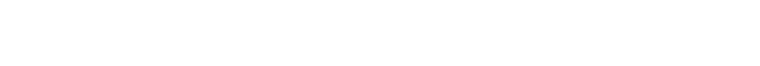
وارفاع الكالف النسوقية ولإنتلجية من ناحية أخرى.

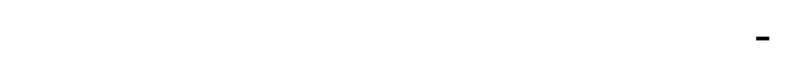

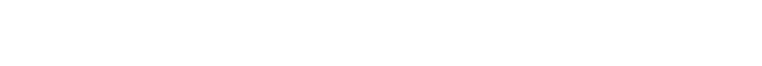

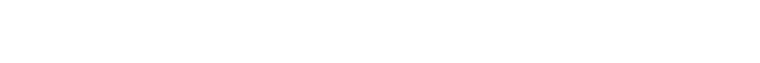

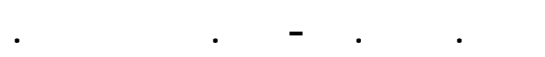

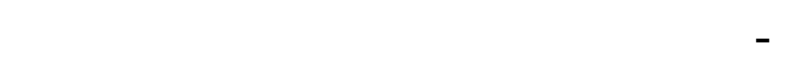

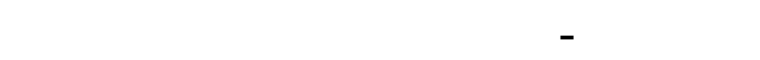

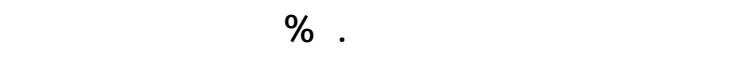

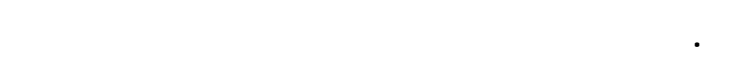

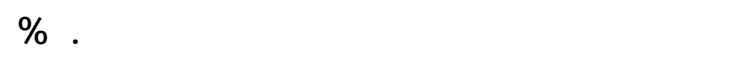

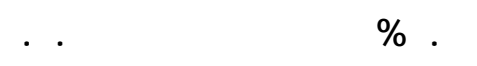
ع -بلغ متوسط مؤشر قوة تصطير البلملس المصرية اللعالم

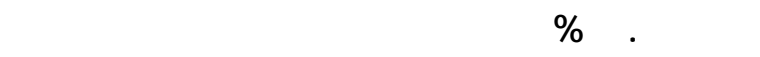

' ققم الاقتصاد وإدارة الأعمل الزراعية، كلية الزراعة، جلمعة الإسكندرية.

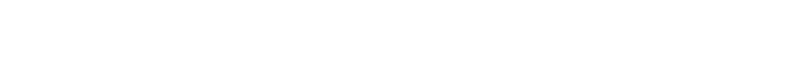

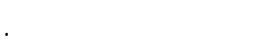

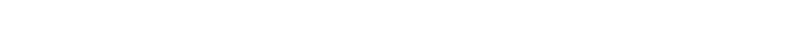




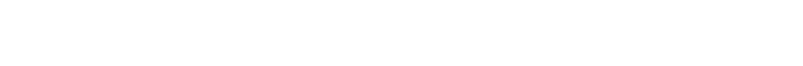

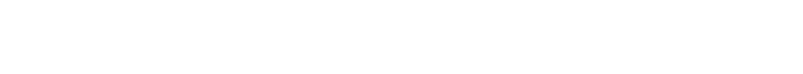

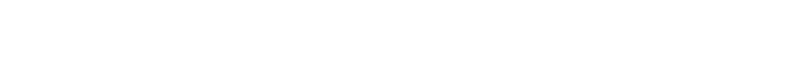

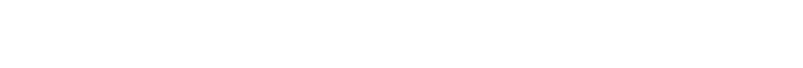

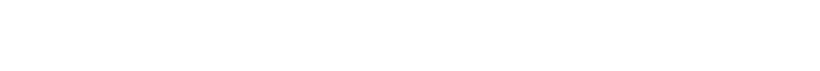

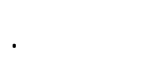

\section{الأهمية البحثية}

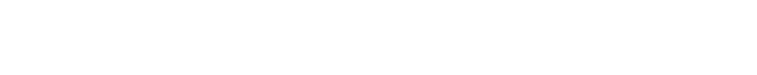

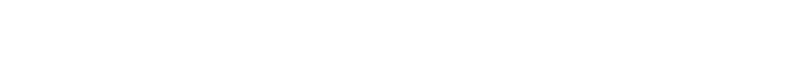

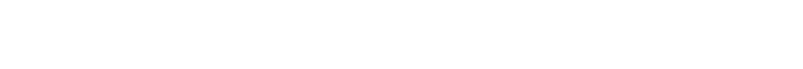
الأسعار أومدي ثبات ولسنقرار الإنتاج، والقدرة على الوفاء لتهاء

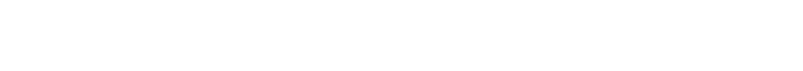
بالدرجة الأوله على كفاءة العملية التصديرية والتي تعتم 2.

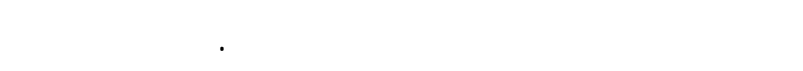

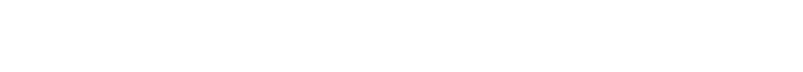

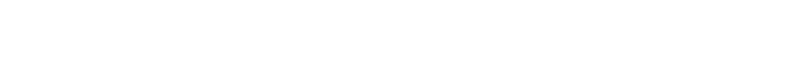

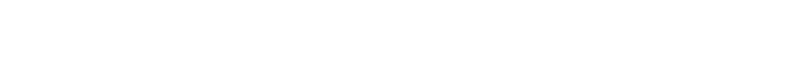

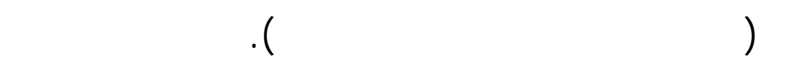

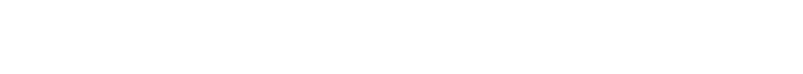

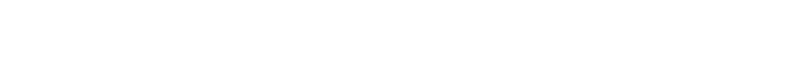

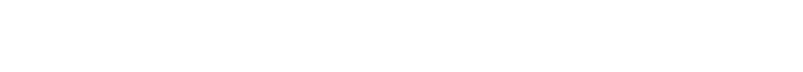

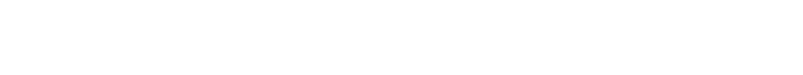

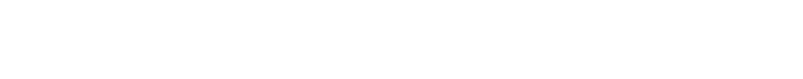

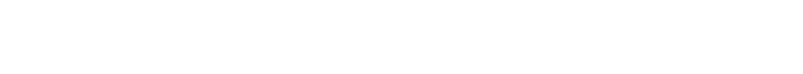

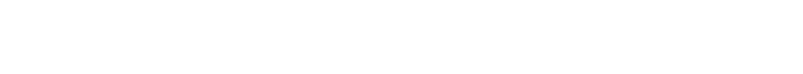

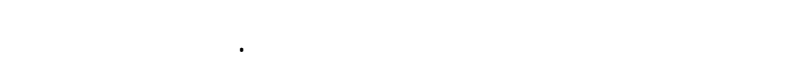

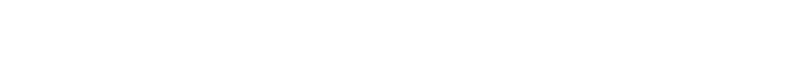
الطبيعية لكن عدم الظوير والتحديث وسوء الإدارة لا يخلق ميزة تنافسية، وهنك دول لخرى لديها ندرة ف في الم ـوارد

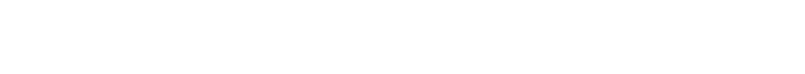

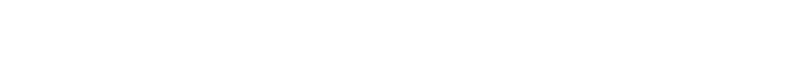

الترتيب، كما بلغ متومط مؤشر معالل عم الإسقرال لمعر

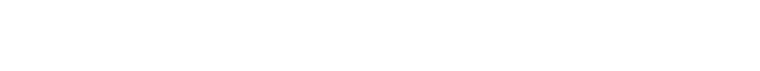
\%r.vr \%r7.rA \% \% \%.ro.v عل الترتيب.

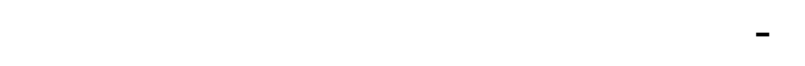
البلملس المصرية للأسواق الإيمالية والروسية واليونلنية

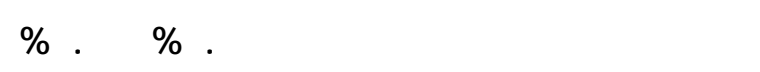

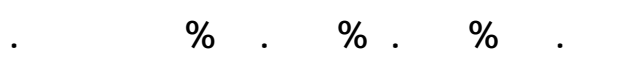

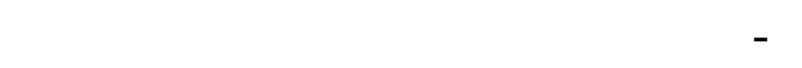

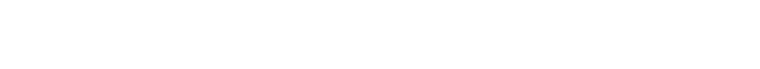

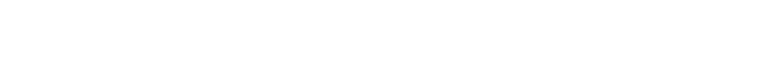
معسعر التصعير المصري، قد ثبتت المعنوبة الإحص الئية

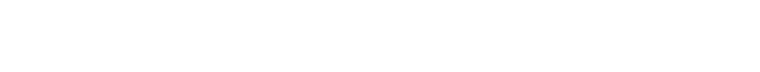

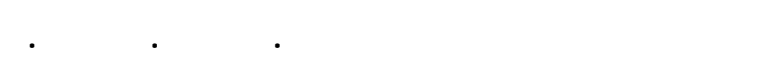

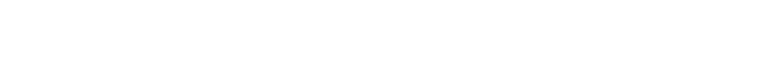

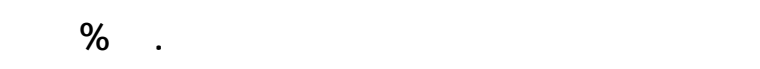

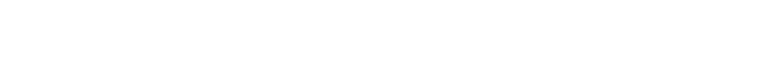

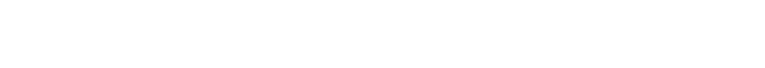
المصرية.

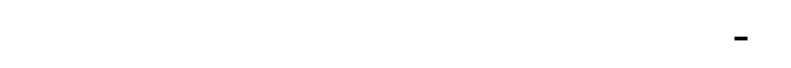

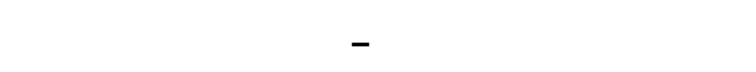

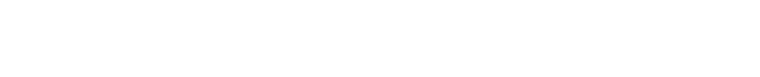

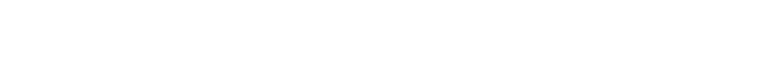

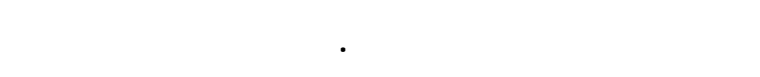

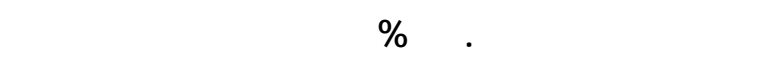
والبالة حوالي I.r.

\section{المقمة والمشكلة البحثي ة}

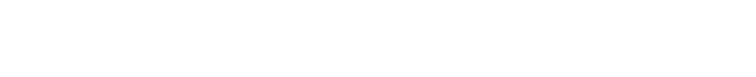

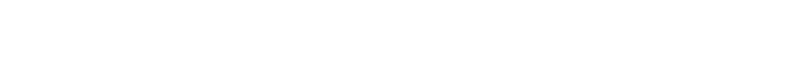

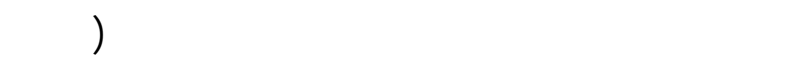

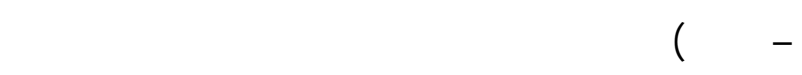

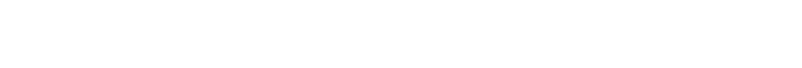
القيلسيه وخلو البططس المصدره من مرض ورك الهن البذه. 
وقيلس الكفاءة التسويقية وكفاءة التجارة الخارجية بلستخدلم بعض المعايير والمؤثرات المتمثلة في التالي:

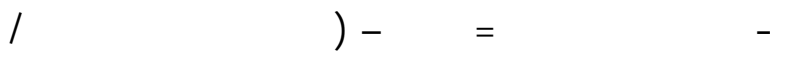

X (التكالف التسوقية + متوسط تكلفة إنتاج الوح ــة)

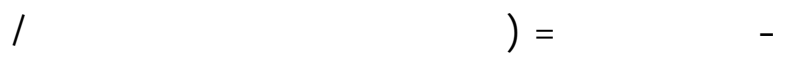

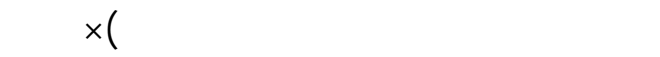

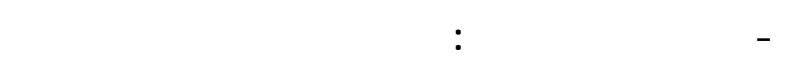
الهنهي للنب المئوية لانحراف القيم المقدرة عن القيم

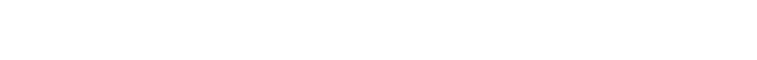
المعلل تساوي صفر وكلما زاد المعلل عن الصفر لل علي زياة عمم الاسنقرار. معلل عمم الاسقرار=

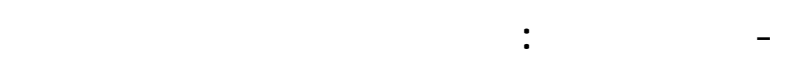

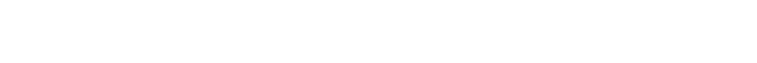

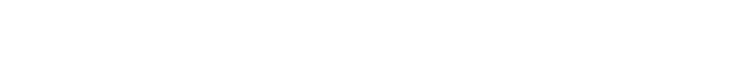
وفة للمعادلة:

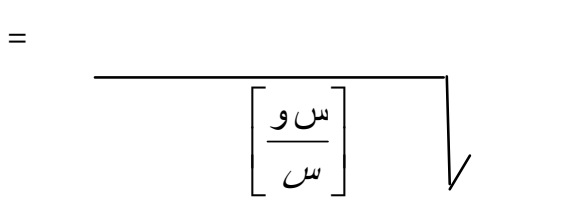
حيثس و: كمية الواردات من الملعة إل ي الدول ة (و).

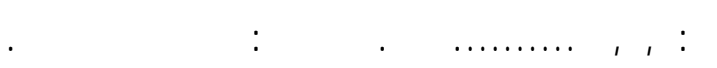
ويصل هذا المعلمل إلي اقصي قيمة له وتن اوي . . في حالة ما إذا كلنت اللسلعة تصدر إليسوق ولحد فتط من الأسواق المستيرادية بينما تقل هذه القيمة كلما توزعت كمية الفية الصادرات علي عدد كبير من الأسواق المستيرادية ، ويري

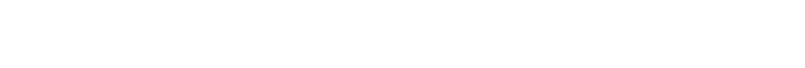

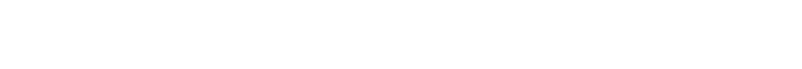
ققلبت كميةشية نسبياً خاصة في قيمة الصادرات.
وإدارة مرنة مما خلق لها قدرة تنافسية عالية. وق 2 إزدادت

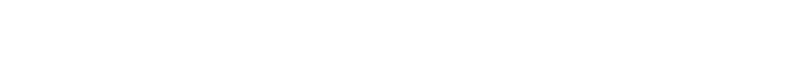

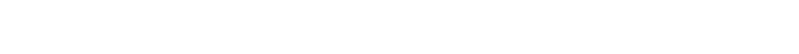

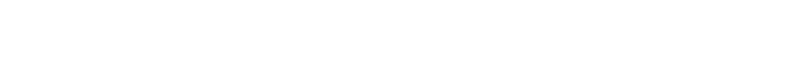

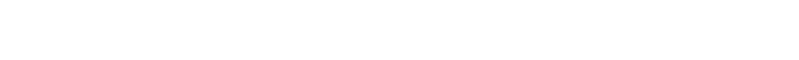
المنافسة بين الهيئت النسوقية المختلفة وتشجيع الدولة لفتح لمسواق جدية والاتجه نحو التصدير. أهرل البهث

يستهف هذا البهث بصفة لملدب ية درلمد مة مح ددات الكفاءة النسويقية، ومحددات صادرات الطلطس المص -رية الطالية من خلل درلمة الأهداف الفرعية التالية: (1) تحليل الهيكل

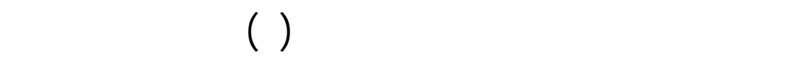

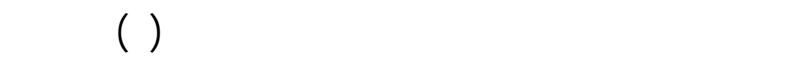

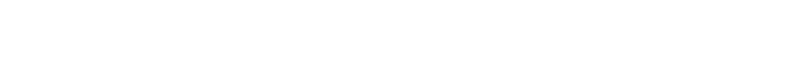

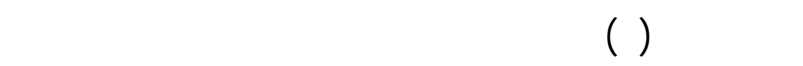

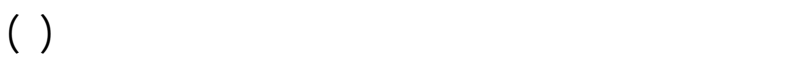

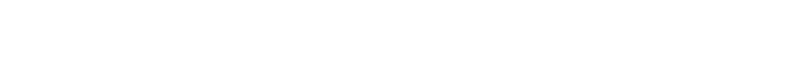

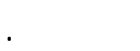

\section{مصادرالبيلنت}

اعتمد البهث بصفة لسلسية للحص ـول عل ه البيان لـ الت

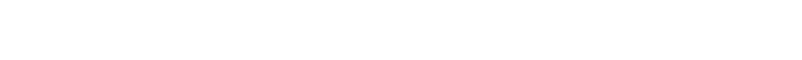

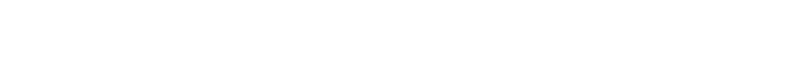

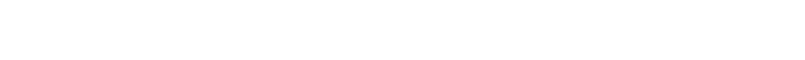

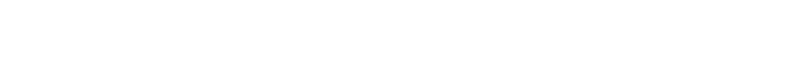

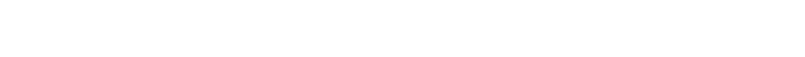
الإبستعنة بعض الأبحلث والدرلسلت اللد القة ذات الص لـة

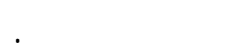

\section{الإبسلوب البحثي}

اعتمد البهث في تققيق أهدافه على إليد لـوب التحلي لل

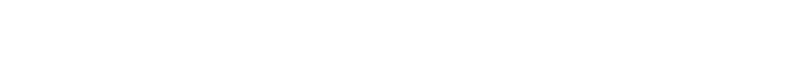

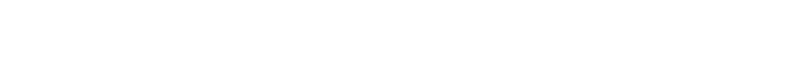


وتتأثر الكاعة التسويقية بتدريج اللسلعة إلي درجلت ورتب

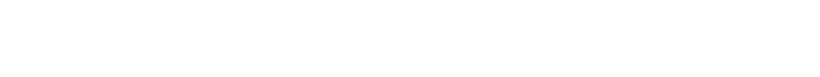

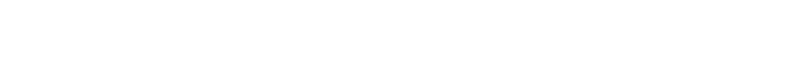

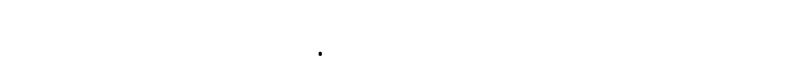

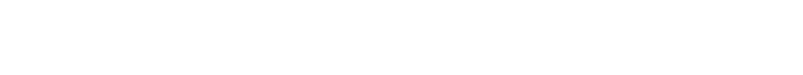

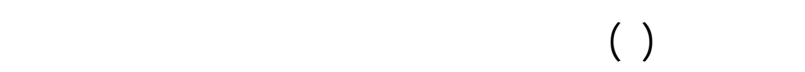
عمليك البيع واللثراء والنولهي اللسعرية في البنيان

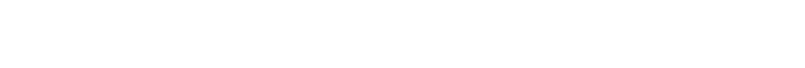

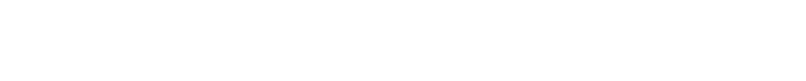

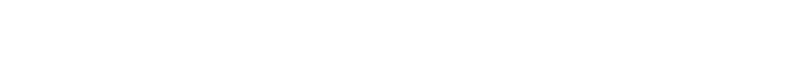

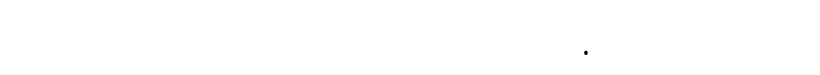
خفض تكالف المخلات أي التكالف التسويقية، بينما تؤوي

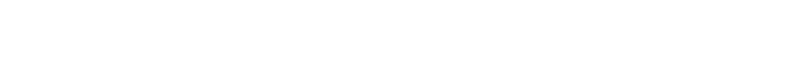

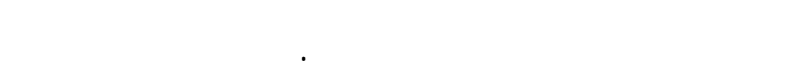

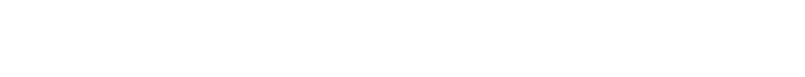
مدي الكفاعة التي تؤدي بها الوظاف النفاضو التسوقية.

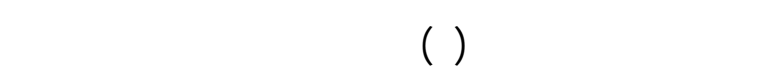

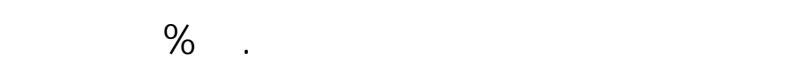

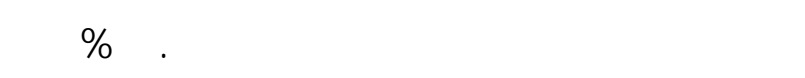

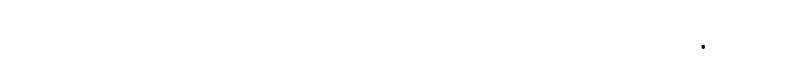
التسويقية لمحصول الططس تبين أنها تكاد تكون ثابتة

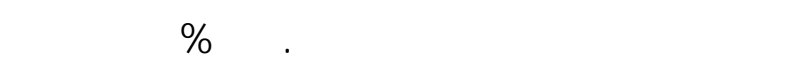

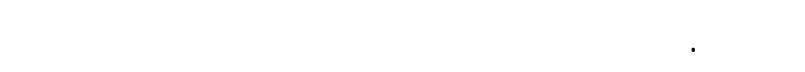

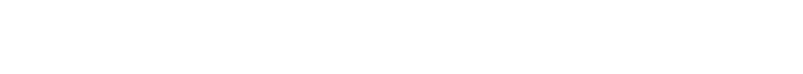

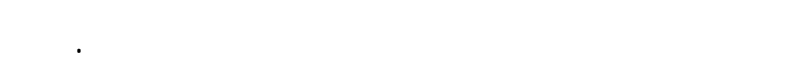
يكون ذلك رلجعاً لانخفاض السعر الدي يحصل عليه المنتج

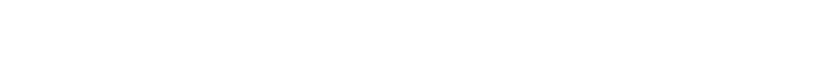

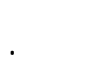

0 - النصيب اللسوق: يعتبر مؤثر النصيب الله -وق لح د 2

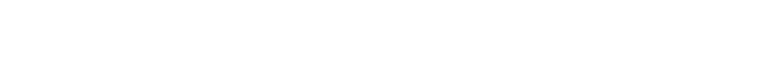

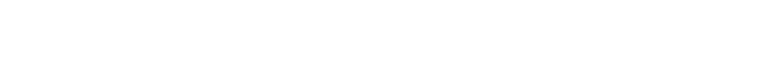

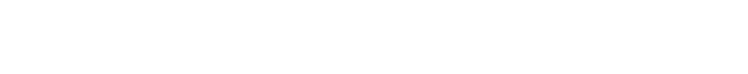

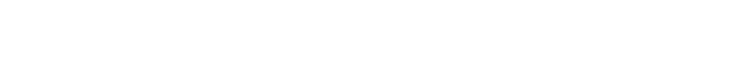

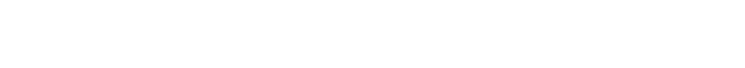

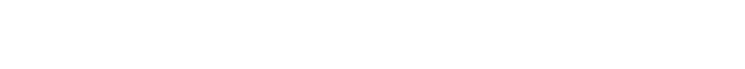

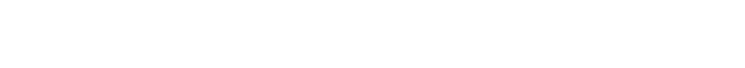

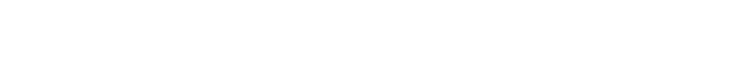

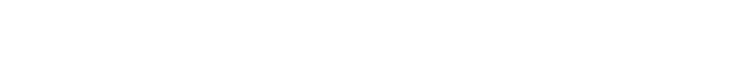
الطلطس المصرية إلى أهم وأعلى الأند -واق العالمي ـة المبنة

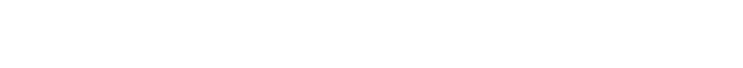

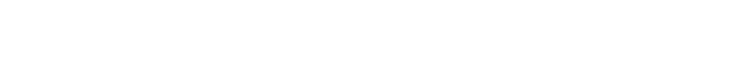

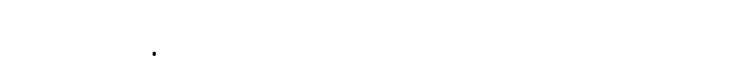
المؤثر يعكس ارفاع الوضع التنفسي لأي دولة.

\section{نتلئج البهث}

\section{ألواًا - قفير الهاءة النسوقية لملهة البللملس:}

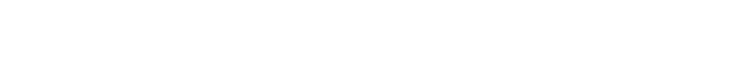

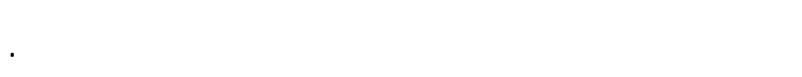

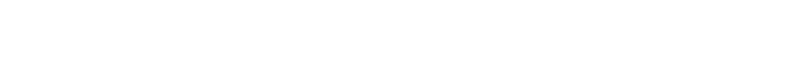

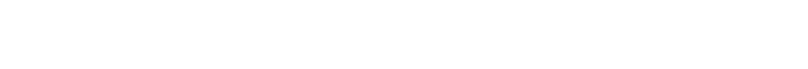
المستهلكين وعناصر الإنتاج المستخمة في الوظاف فلف

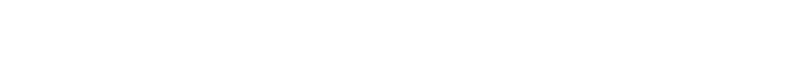
ومعبراً عنها بتكالف أداء الوظايف التسويقية.

تقنم الكفاعة التسويقية إلي قسمين هما: (1)الفائة

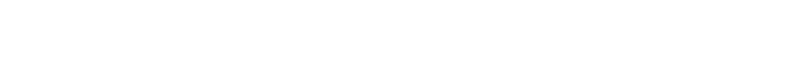
لققليل مقدار التكالف اللازمة للثل أو التخزين أو التوصل التوطي

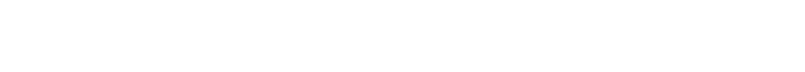
(الجول بالنسة اللططس (مما تمل نسبة التاف أثناء الثل. 
جنط رقم ا. الكالف الإنتلجية الكلية والهاءة النسوقية لمحصط البململس خلال الفترة (1994 - rا - r).

\begin{tabular}{|c|c|c|c|c|c|}
\hline 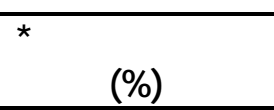 & التكالف النسول/لنية) & تكلة إنتاج / الملالن & 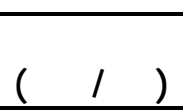 & (جكلهة النتاج / الفدل) & السنة \\
\hline $7 \cdot . \mu$ & $r \varepsilon\rceil$ & $r V \mu .7$ & $9 . \varepsilon$ & $r 0.0$. & 1991 \\
\hline $0 \Lambda . \varepsilon$ & $r 07$ & r09.1 & 9.1 & rorl.7 & 1999 \\
\hline$\mu \varepsilon .7$ & 711 & $\mu \Upsilon \mu . \varepsilon$ & 9.9 & भ\૧દ. & $r \cdots$ \\
\hline${ }^{\mu} \wedge . V$ & ह१। & н.9.7 & $1 \cdot . \cdot$ & $\mu 1 \cdot 0 . r$ & $r \cdot .1$ \\
\hline$\varepsilon \Lambda .9$ & rov & нEr. 7 & $1 \cdot .1$ & 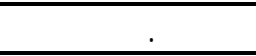 & $r \cdot r$ \\
\hline$\mu 7 . \varepsilon$ & TVO & $\mu \wedge 0.0$ & $1 \cdot . \mu$ & чq८7. & $r \cdot \mu^{\mu}$ \\
\hline HV. & $\Lambda \cdot V$ & $\varepsilon V \varepsilon .7$ & $1 \cdot . \mu$ & EAVT.O & $r \cdot \cdot \varepsilon$ \\
\hline 01.0 & EOr & $\varepsilon \Lambda \cdot .0$ & $1 \cdot .0$ & 0.71 .0 & $r \cdot .0$ \\
\hline${ }^{\mu} \wedge . \Lambda$ & $\Lambda V$. & 001.1 & $1 \cdot .0$ & $O \vee \wedge \Lambda . \cdot$ & $r \cdot \pi$ \\
\hline E7.Y & VYI & 719.1 & $1 \cdot . V$ & $770 \cdot$. & $r \cdot \cdot v$ \\
\hline$\varepsilon 7.7$ & $\Lambda \mathrm{OH}^{\mu}$ & VER. 9 & $1 \cdot .9$ & $\Lambda \cdot 9 \% .0$ & $r \cdot \cdot \Lambda$ \\
\hline$\varepsilon \cdot .0$ & 11.1 & $V \varepsilon \wedge .9$ & 11.1 & $\Lambda \mu \| .0$ & $r \cdot . q$ \\
\hline$\mu .0$ & 1790 & 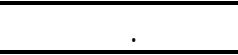 & $1 \cdot .9$ & $\Lambda \cdot \Lambda 7.0$ & r.1. \\
\hline rI.V & 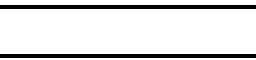 & $V \wedge \Lambda .9$ & 11.1 & AVOA.r & $r .11$ \\
\hline $1 \Lambda .1$ & $\mu 97 \mu$ & $\Lambda \vee \varepsilon . \Lambda$ & $11 . \mu$ & 9170.1 & $r \cdot I r$ \\
\hline$\varepsilon \cdot .0$ & $1.7 r . \Lambda$ & $0 \varepsilon 1 . \mu$ & $1 . .0$ & $0 \mathrm{~V} 0 \cdot .0$ & |المتوبط \\
\hline
\end{tabular}

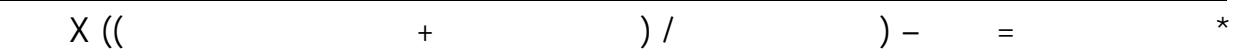
المصدر: جُمعت ومُسبت من وزارة الزراعة ولستصلاح الأراضي، قطاع للشئون الاقتصاية، الإدارة المركزية للاقتصاد الزراعي، شأشرة الاقتصاد الزراعي، أعداد مفرقة.

ع =عسعر التجزئة للطن من الططلس.

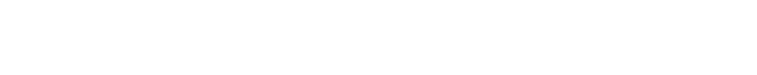

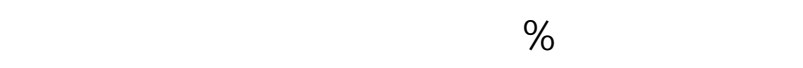

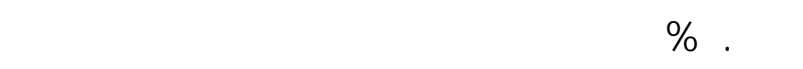

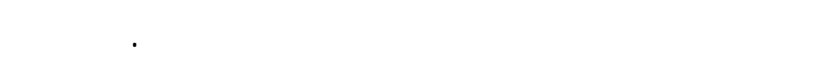

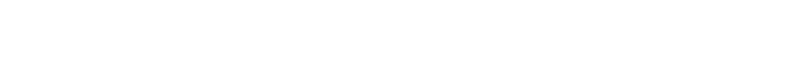

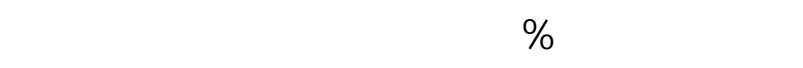

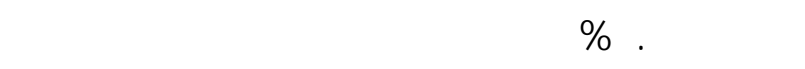

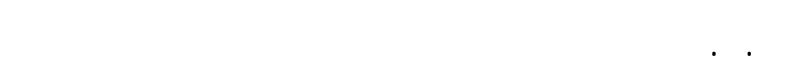

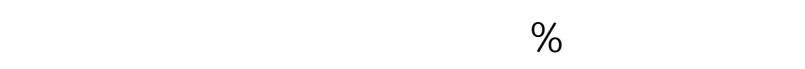

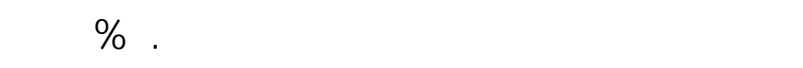

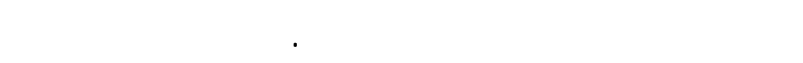
المحددات الأخرى ثابتة عند مستوى معين.

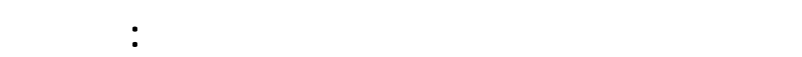

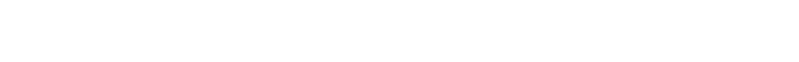
وفقاً للنموذج الرياضي كما يلي:

$$
\begin{aligned}
& \text { لوص = }
\end{aligned}
$$

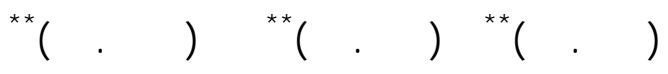

$$
\begin{aligned}
& { }^{* *}(00.7 \mathrm{~V}-) \\
& \text { ف* } \\
& .99 \mathrm{~V}={ }^{r-} \text {, }
\end{aligned}
$$$$
\text { r. }
$$

حيث: ص = الكفاءة النسويقية. عه = = اللسعر المزرعي للطن من الطلطس. تين = تكالف انتاج الطن من الططس. 
I 1 ·r، ولم تثبت معنوية معل النمو للسنوي إحصائياً.

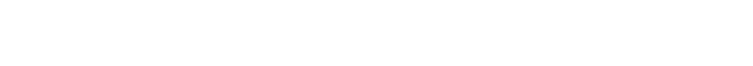

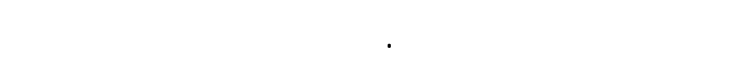

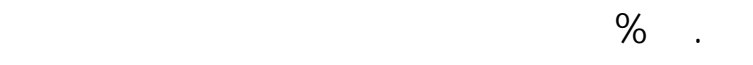

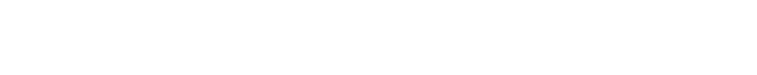

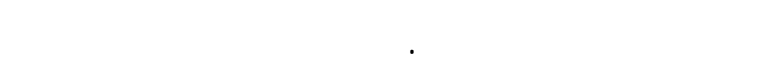

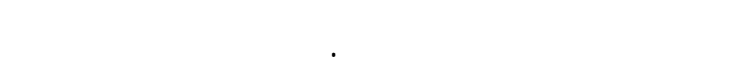
I 1 -r، ولم تشبت معنوية معدل النمو السنوي إحصائيًا -

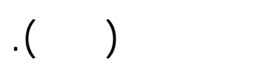

r - السوق اليونافي: لحل للسوق اليونانى المرتبة الثالثة

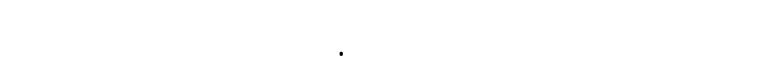

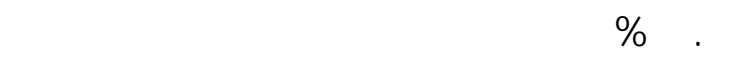
الططس خلل متوسط فترة الدرلسة، تبين أنها

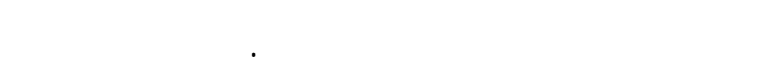

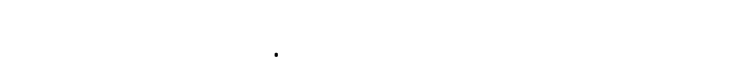
ع · · r، ولم تثبت معنوية معل النمو للسنوي إحصائيً. وقد بلغت قيمة الصادرات المصرية من الططلس إلى

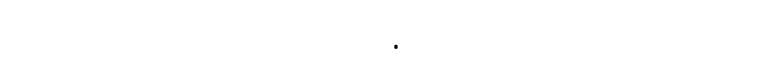

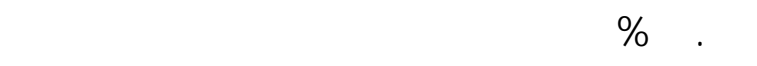

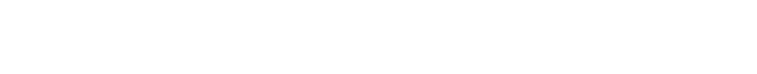

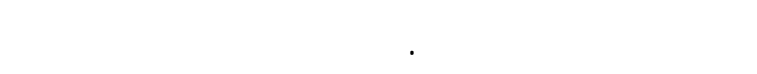

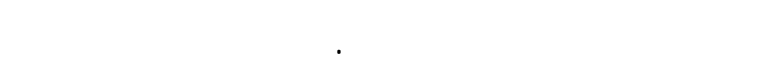

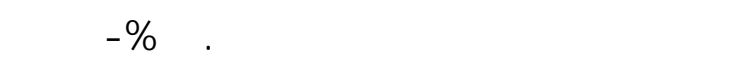
رقم (r, ع- اللسوق الأماني: لحل للسوق الألمافى المرتبة الرالبعة

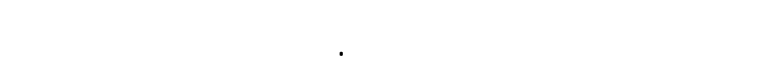

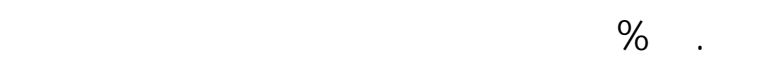
الططس خلل متوسط فترة الدرلسة، تبين أنها

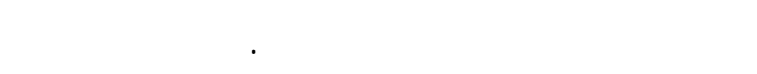

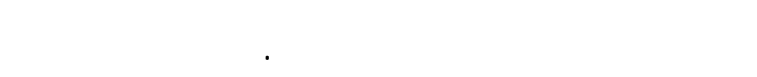

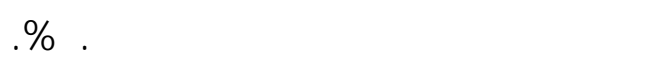

\section{ثلنياً - التسوق الخارجي لإبلاس المصربة:} أ - التوزع الجغراهي لصارك المالبلس المصربة لأه م الأسواق الهالمية:

يتناول هذا الجزء من الدرلسة تحليل ظور التوزيع الجغرافي لصادرات الططس المصرية إلى دول العالم

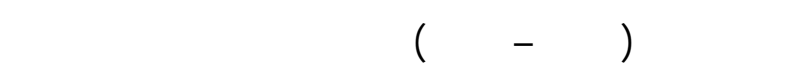

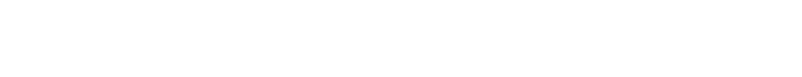

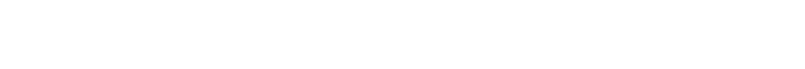
\% من متوسط كمية صادرات الططس المصرية وذلك على النحو التالى:

ا - السوق الإيلال: لحل السوق الاطاله المرتبة الأوله

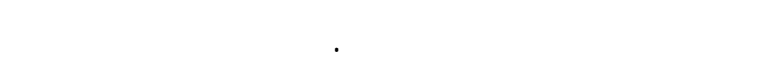

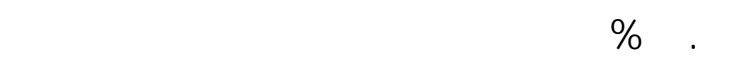

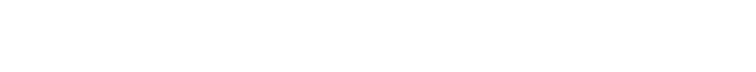

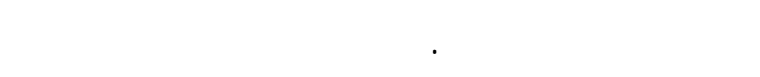

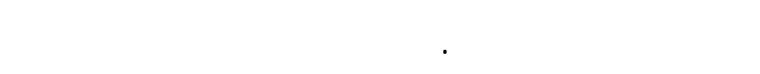

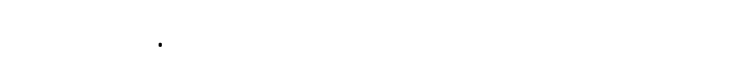

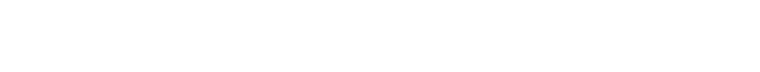

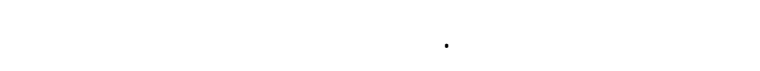
\% I A.OV

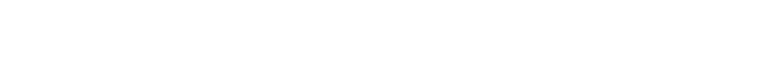

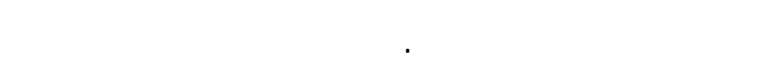

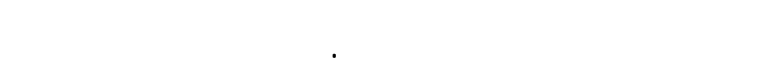

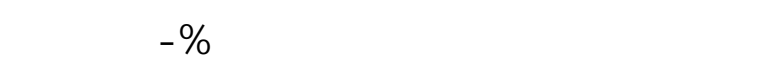

r - اللسوق الروسي: لحل السوق الروسه المرتبة الثانية

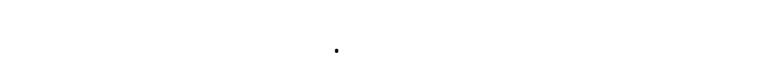

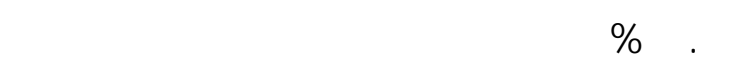
البطلس خلل متوسط فترة الدرلسة، تبين أنها

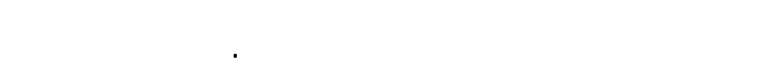

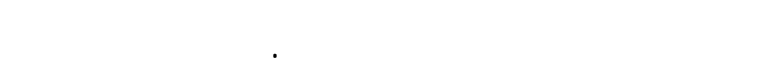




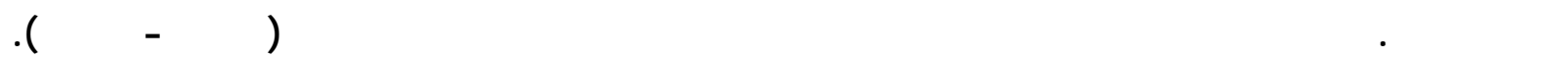

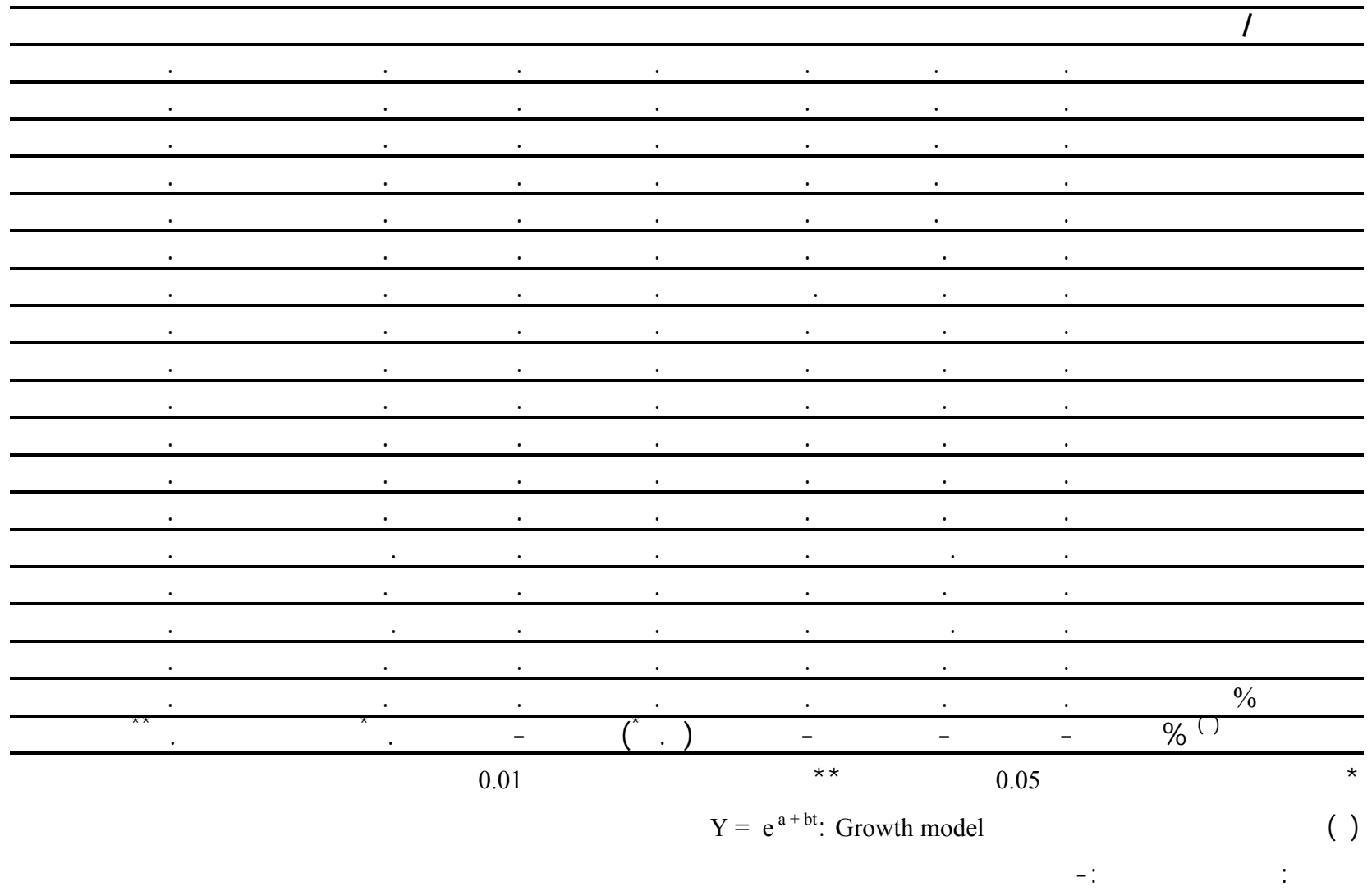

ا - الجهاز المركزي للتعبئة العلمة والإحصاء، قاعة بيانت التجارة الخارجية "مصر إنترنت"، بيانت غيرمنشورة، الموقع الرسمي

.www.capmas.gov.eg

r - الجهاز المركزي للتعبئة العلمة والإحصاء، الشرة للسنوية للتجارة الخارجية، أعدادمختلفة.

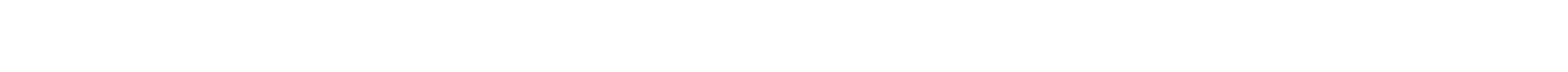

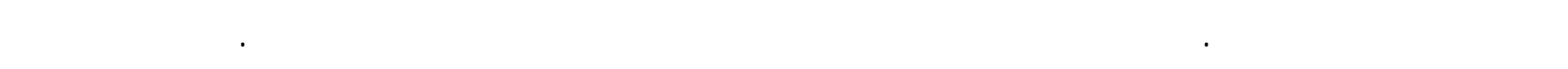

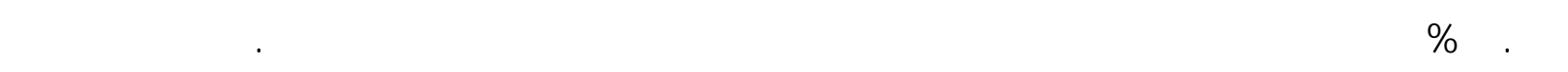

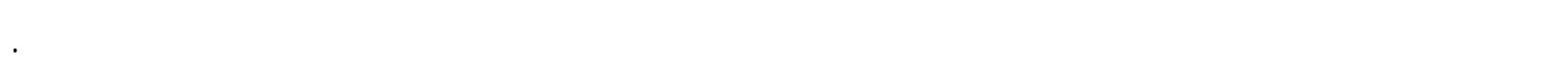

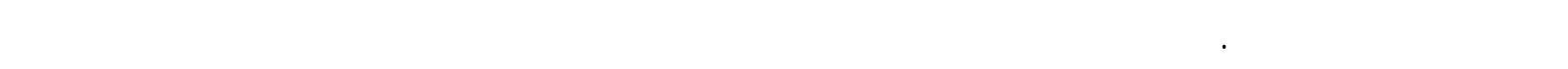

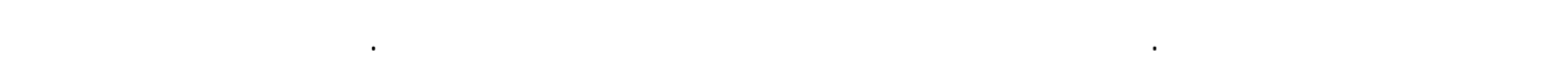

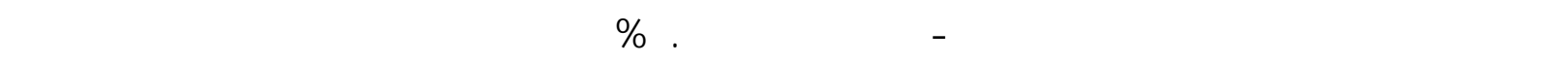

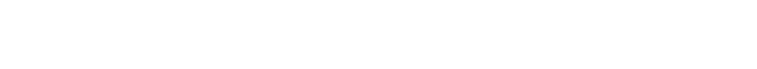

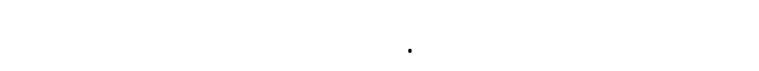

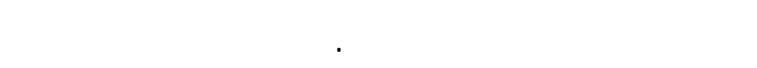

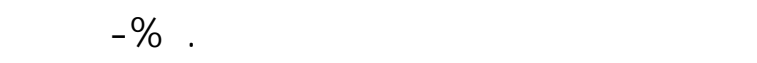
رقم (r, r).

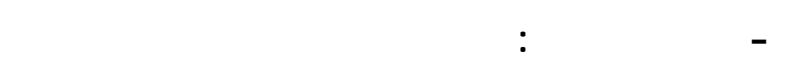

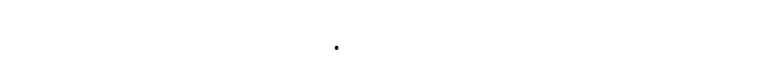

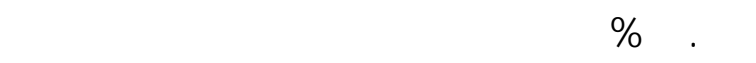




\begin{tabular}{|c|c|c|c|c|c|c|c|}
\hline إجمالي صاكركات مصر & دل لخرى & لبنل & الملنيا & اليونلن & תפטרו & الي & المنة /الدولة \\
\hline 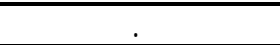 & $1 V . \cdots$ & $0 . \cdot 1$ & $1 \cdot .1 \varepsilon$ & V. $\varepsilon \varepsilon$ & $\because \cdots$ & r.07 & 1991 \\
\hline$\varepsilon 7^{\prime} \cdot \mu$ & $10 . \mu \Lambda$ & $\mu . \mu$ & 14.91 & $\mathrm{~V} . \cdot \Lambda$ & $\because \cdots$ & $7 . r \Lambda$ & 1999 \\
\hline r.7.77 & 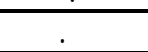 & E.VO & $0.7 \Lambda$ & r.qu & $\therefore \cdots$ & $0 . \Lambda Y$ & $r \cdots$ \\
\hline rq.Vo & V.A7 & 0.7 & V.E. & 1.19 & $\therefore \cdots$ & V.Or & $r \cdots$ \\
\hline$\varepsilon r .7 r$ & $1 \cdot . \cdot 1$ & $\varepsilon . \wedge \vee$ & А.हV & I. ${ }^{\mu} \mathrm{V}$ & $\cdot . \mathrm{Y \Lambda}$ & $\Lambda .0 \varepsilon$ & $r \cdot r$ \\
\hline$\varepsilon^{\mu} .9 V$ & $\mathrm{~V} . \cdot \mu$ & 0.7 & 0.17 & $\Lambda .0 \Lambda$ & 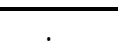 & $11 . v$. & $r \ldots \mu$ \\
\hline $7 V . \cdot 7$ & $\overline{\mid \varepsilon .7 \mu}$ & 0.17 & $1 \cdot .7 \mathrm{~V}$ & rI.I. & E. 10 & $1 \cdot .70$ & $r \cdots \varepsilon$ \\
\hline VV.11 & IT.MO & V.10 & 17.17 & $19 . \wedge \mu$ & $0 . \cdot \mathrm{V}$ & $10.0 \varepsilon$ & $r \cdot 0$ \\
\hline $7 \varepsilon .91$ & 9.10 & $r . \varepsilon r$ & $1 \cdot .90$ & 10.77 & १.ह. & IV.YY & $r \cdots \tau$ \\
\hline $1 \cdot 0 . V$. & 19.47 & r\%.Vq & 9.99 & 10.7 & $1 \% .7 \Lambda$ & YY.YN & $r \cdots V$ \\
\hline $17 \mu .1 \mu$ & " & $|\nabla . \varepsilon|$ & $7 . r 1$ & $\mu 1.9 \mu$ & YV.ET & हץ.77 & $r \cdots \Lambda$ \\
\hline 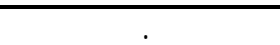 & I I I I I & 10.r. & rา.Vo & $\mu \cdot \mu l$ & $19.0 \varepsilon$ & $r \cdot . \varepsilon r$ & $r \cdots q$ \\
\hline $1 \mu^{\mu} \cdot .7 \Lambda$ & זА.КА & $\Lambda . \mu \varepsilon$ & 10.17 & $r 0.7 \Lambda$ & ro.ro & YV.AM & $r \cdot 1$. \\
\hline$r O \cdot .00$ & $00 . \mu l$ & V. ${ }^{\mu V}$ & $1 \% .99$ & $r V . \cdot V$ & $11 \mu . \mu \mu$ & 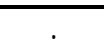 & $r \cdot 11$ \\
\hline IY7.V" & rA.0E & 11.19 & YY.97 & 17.99 & r१.१0 & IV.Y. & $r \cdot 1 r$ \\
\hline$r \cdot 0.0$. & $\overline{O V .} \cdot \mathrm{V}$ & Үץ.हq & 7.79 & YT.YO & $09 . \varepsilon \mu$ & 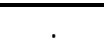 & $r \cdot \mu^{\mu}$ \\
\hline $9 \Lambda . \cdot V$ & YY.OY & $9 . \mu \varepsilon$ & $I T \cdot \cdot r$ & 17.00 & $19 . \varepsilon \varepsilon$ & $|\Lambda . Y|$ & المتوطي \\
\hline $1 \cdots$, & rY.97 & $9.0 r$ & IT.YO & $17 . \Lambda 1$ & $19 . \wedge r$ & $11.0 \mathrm{~V}$ & $\%$ \\
\hline "* $1 \mu . \varepsilon$ & $11 . \mu$ & 9.0 & - & $1 \% .1$ & - & $1 \varepsilon$ & معدل النمو (1)\% \\
\hline
\end{tabular}

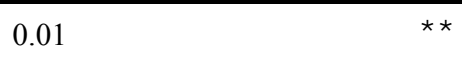

0.05 * معنوي عند المستوى الاحتمالي

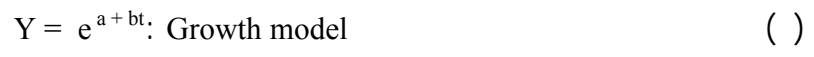

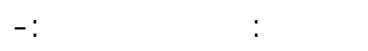

ا - الجهاز المركزي للتعبئة العلمة والإحصاء، قاعة بيانات التجارة الخارجية "مصر إنترنت"، بيانت غيرمششورة، الموقع الرسمي

.WWw.capmas.gov.eg

r - الجهاز المركزي للتعبئة العلمة والإحصاء، النشرة اللسنوية للتجارة الخارجية، أعدادمختلفة.

\section{ب - المؤشرات والمحدات الفتصاية لصالرات البمللس \\ المصربة:}

ا. المسلحة المزروعة من الططلس المصرية: تراوح عت

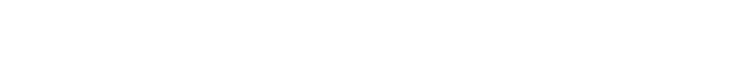

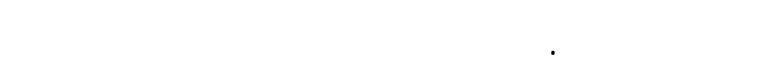

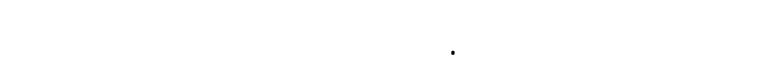

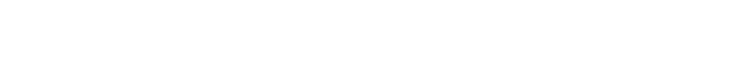

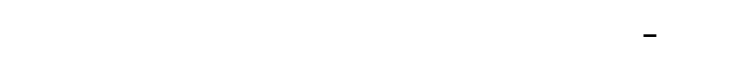

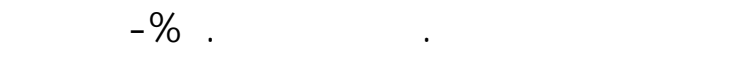

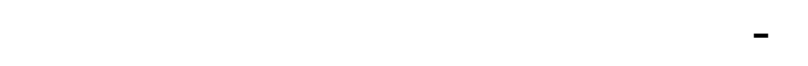

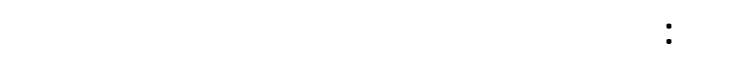

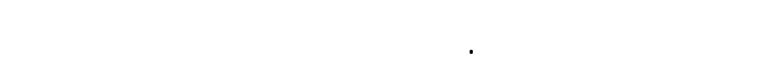

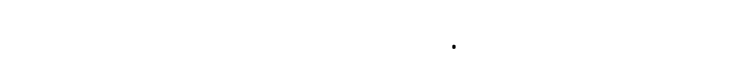

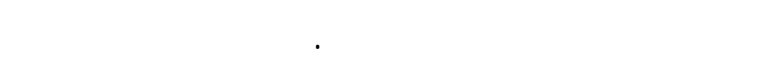

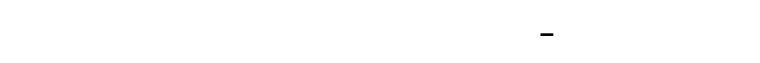

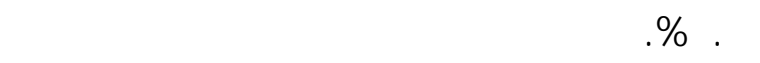

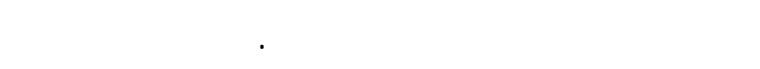

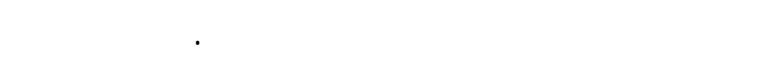

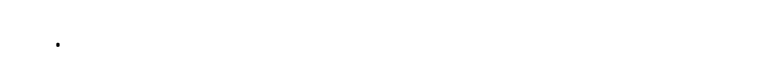

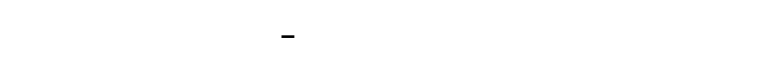

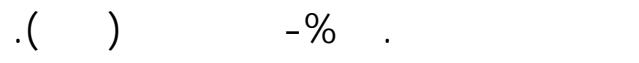


rا ·r، وبمعل نموسنوي عند مستوى معنوية I . . بلغ نحو r.\% - جدول رقم(ع).

\section{ع -مؤشرقوة تصسير البالملس المصرية:}

قيلس قوة تصدير الططس المصرية للعالم تبين أن هـ

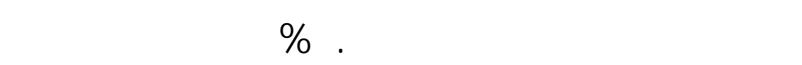

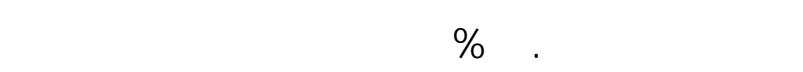

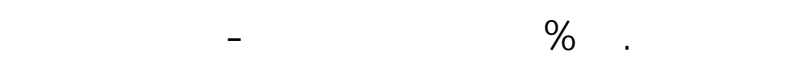

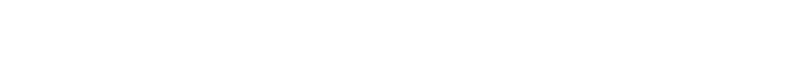

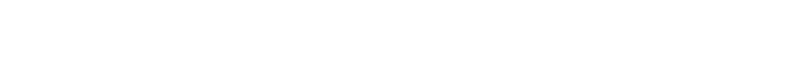

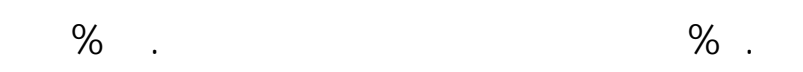

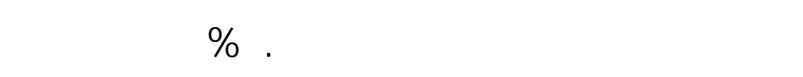

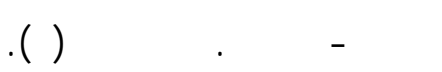

r. الإنتاج المحلى من الطلطس المصرية: تراوح الانتاج

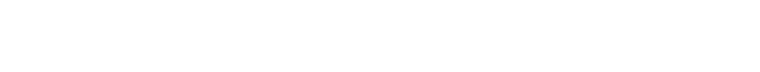

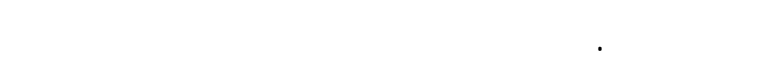
بلغ حوالي ع ·. .

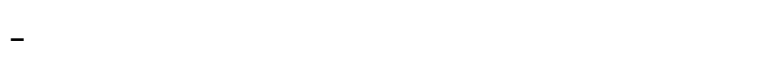

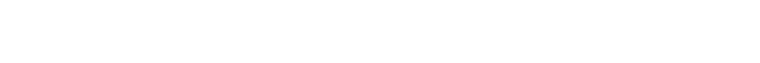

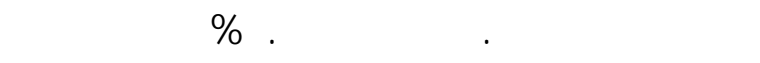
الدرلسة - جدول رقم(ع). ". الاستهلاك المحلى من الطلطس المص -رية: تراوح ـت

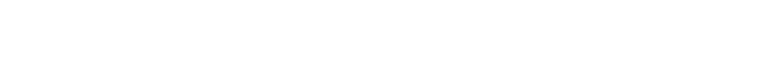

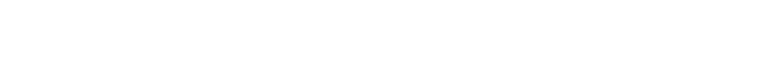

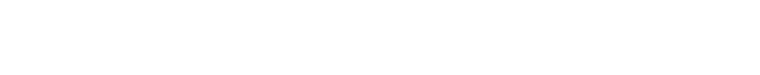

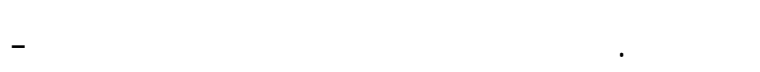

جطل رقم ع. قلورطساحة ولنتاج ولستهلاs وقوة تصسير البالملس المصرية خلال الفترة (199 1 -مו - م).

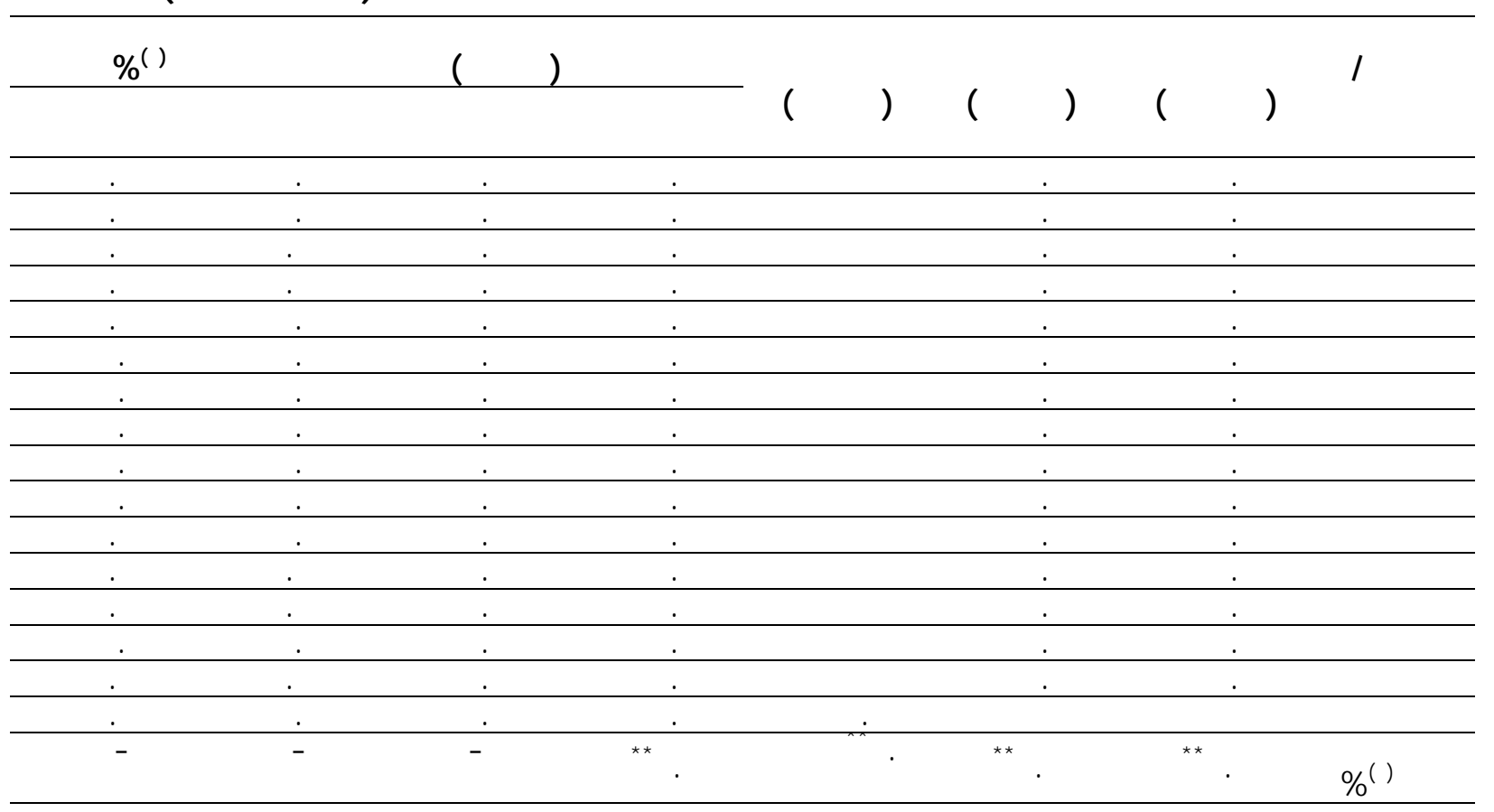

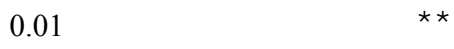
* معنوي عند المستوى الاحتمالي 0.05

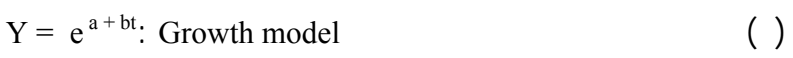

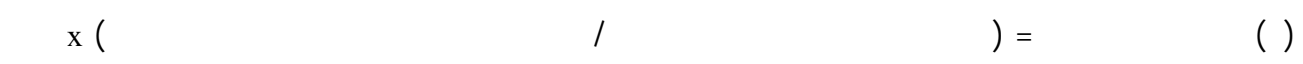
المصدر: جمعت ومسبت من: - (المون ا - وزارة الزراعة ولستصلاح الأراضي، قاعاع اللشئون الاقتصادية، ششرة الإحصاءات الزراعية، أعداد مختلفة، القاهرة. r - وزارة الزراعة ولستصلاح الأراضي، قاعاع اللشئون الاقتصاية، نشرة درلمة الماعية الميزلن الغذائي بجمهورية مصر العربية، أعداد مختلفة، القاهرة. 


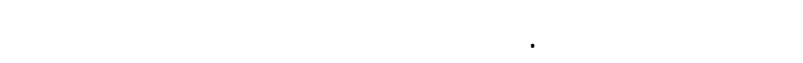

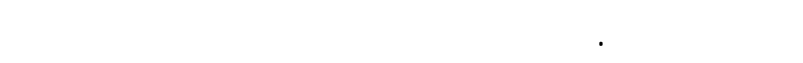

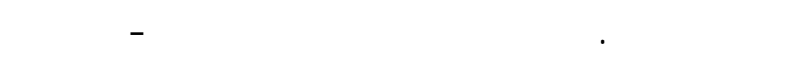

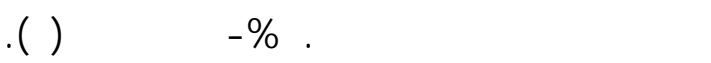

\section{V - نسبقسعر التصطير المصري للسعر الهالي:}

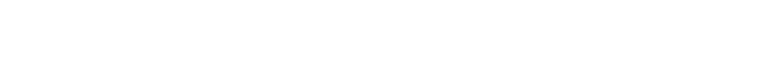

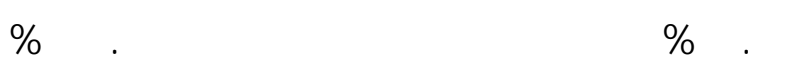

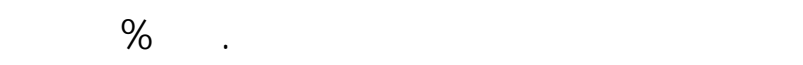

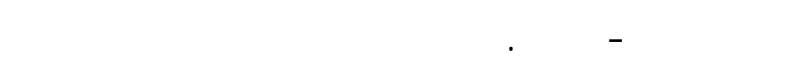

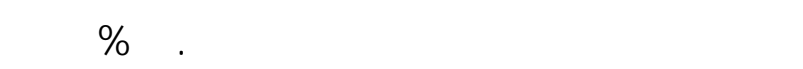

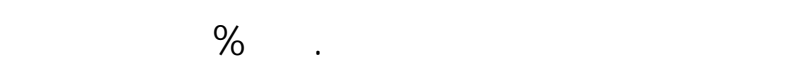

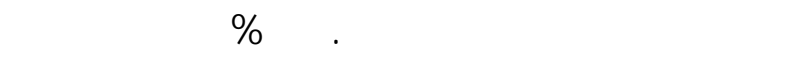

1991 - rו •r - جدول رقم (0).

\section{0 -مسحر التصير المصري للان:}

تراوحسعر التصير المصرى للعالم لطن الططس بين

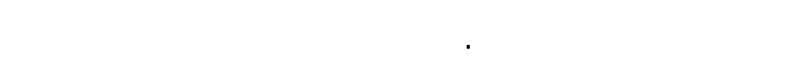

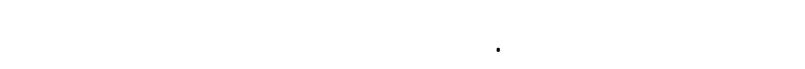

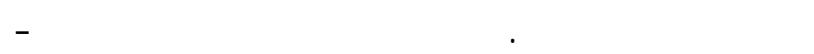

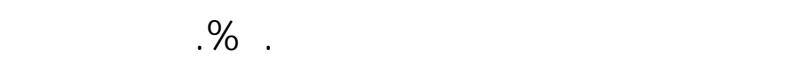

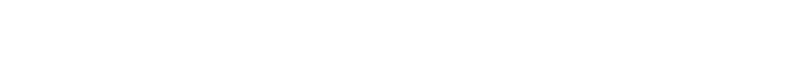

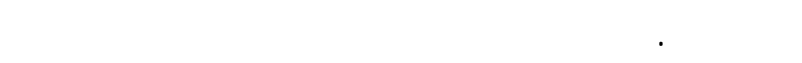

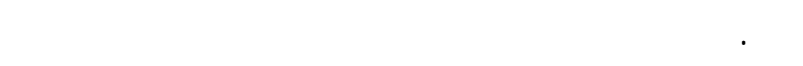

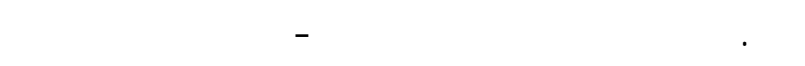

سنوي بلغ نحو \%. \% - جدول رقم (0).

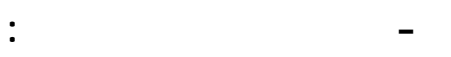
تراوحسعر التصدير العالمي لطن الططس بين حد

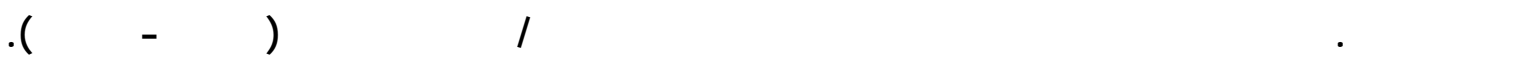

\begin{tabular}{|c|c|c|c|c|c|}
\hline \multicolumn{2}{|c|}{ نسبقسعر التصير المصري للسعر } & \multirow{2}{*}{ (رولالمالمبل) التصير) } & \multicolumn{2}{|c|}{ سعر تصدير الجملالس المصربة } & \multirow{2}{*}{ السنة / المؤشر } \\
\hline ل للعالم & لألعال الأسواق & & ل اللعالم & لألي الأسوقة & \\
\hline $9 \cdot . \cdot \mu$ & 11.17 & $r \mid \cdot . \mu$ & 119.19 & $1 \mathrm{~V} \cdot .00$ & 1991 \\
\hline V^.এI & VE.E. & TYA.OE & $I \Lambda \cdot . I Y$ & $I V \cdot . \cdot \varepsilon$ & 1999 \\
\hline 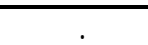 & $9 \Lambda . \cdot r$ & $177.1 \varepsilon$ & $18 \cdot .19$ & $17 \Gamma . \wedge 0$ & $r \cdots$ \\
\hline $9 \cdot . \Lambda \cdot$ & $\overline{\Lambda \varepsilon .7 \varepsilon}$ & |V7.7I & $17 \cdot . \mu \mathrm{V}$ & 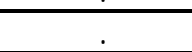 & $r \cdots 1$ \\
\hline 94.99 & $\Lambda 9 . \wedge$. & 199.1. & $110 . V 9$ & IV৭.ह" & $r \cdots r$ \\
\hline VY.Vq & VY.El & $r \cdot \mu . \Lambda \Lambda$ & 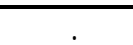 & TEV. Tr & $r \ldots \mu$ \\
\hline$V Y . \cdot \varepsilon$ & $79.9 \%$ & 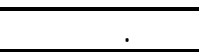 & $1 V 0.91$ & $I V \cdot . V^{\mu}$ & $r \cdots \varepsilon$ \\
\hline $9 \mu .9 r$ & $9 \mu .79$ & $\Gamma \mid \cdot{ }^{\mu} r$ & $19 V .0 \varepsilon$ & $19 V . \cdot \varepsilon$ & $r \cdots 0$ \\
\hline 74.91 & TI.r. & rVV.7V & IVV.EO & IVO.0r & $r \cdots \tau$ \\
\hline$\Lambda \varepsilon . \varepsilon 0$ & NO.ro & rTV.rV & rVר.ह0 & rVq.·V & $r \cdots v$ \\
\hline IYY.rר & $I^{\mu} \cdot r$. & גז.ז" & $\varepsilon \mu q . \varepsilon V$ & $\varepsilon^{\mu}{ }^{\mu} .7^{\mu}$ & $r \cdots \Lambda$ \\
\hline rro.9A & TrE.99 & rq१.н" & 7V7.हع & 7Vץ.ह7 & $r \ldots q$ \\
\hline ITV.. & $1{ }^{1 \mu Y .90}$ & 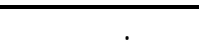 & $\varepsilon \mu\urcorner . \mu 0$ & $\varepsilon r^{\mu} . \varepsilon 7$ & $r \cdot 1$. \\
\hline $1 \cdot 7 . \mu \mathrm{Y}$ & $1 \cdot 0 . \varepsilon 7$ & rVI.10 & एवह.09 & 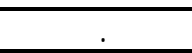 & $r \cdot 11$ \\
\hline IIE.0. & $11 \Psi . \Lambda \varepsilon$ & $\mu^{\mu} V^{\mu} . \varepsilon V$ & $\varepsilon r V .7 \varepsilon$ & $\varepsilon r 0.10$ & $r \cdot \| r$ \\
\hline $1 \cdot \mu . \Lambda \Lambda$ & $1 \cdot 1 . \mu \mu$ & אד.זרז & rAT.rq & rV7.07 & المتوطul \\
\hline \multirow[t]{2}{*}{ T. } & ${ }^{\prime} \varepsilon$ & $0 . \mu$ & ${ }^{n+1} \Lambda . \Lambda$ & $9 . \mu$ & معل النمو \%") \\
\hline & & \multicolumn{2}{|c|}{ **معنوي عند المستوى الاحتمالي 0.01} & تتمالي 0.05 & * معنوي عند المستوز \\
\hline
\end{tabular}




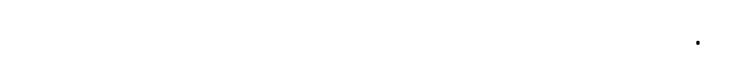

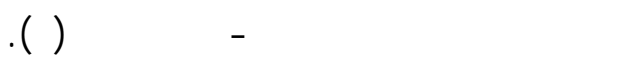

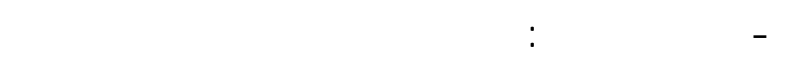

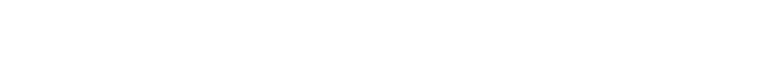

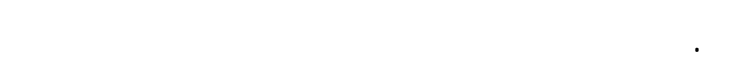

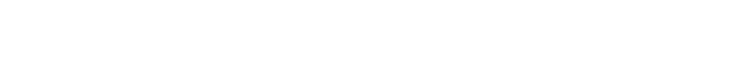

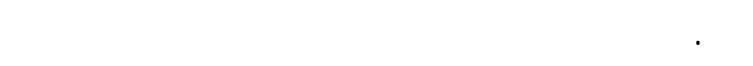

بلغ نحو ؟. \% - جدول رقم (7).

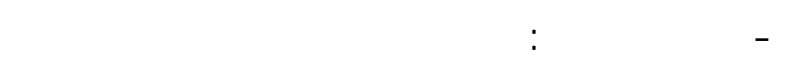

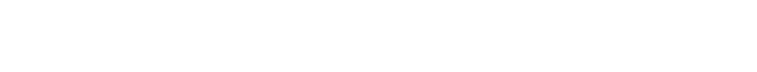

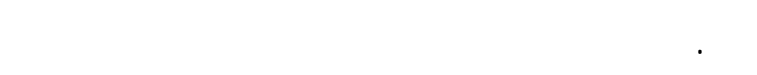

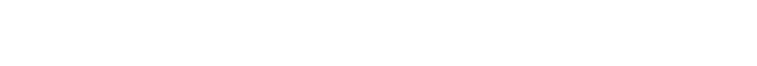

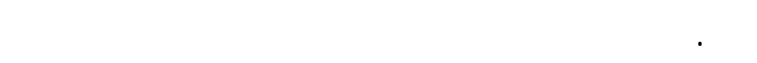

سنوي بلغ نحو ع.9\% - جدول رقم (7).

\section{1 - أسعار تصطير اللانمن البلملس لأهم وأعل لأسوق} العالمية:

I - للسوق الإيطالي: تراومت لـ عارط ن الب لطس

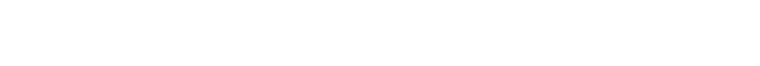

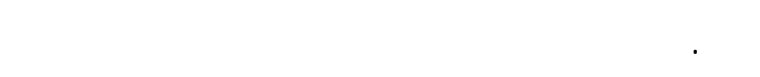
عץ.ع عادولار عل 9 . . r، وبمتوسطسنوي بلغ حوالي

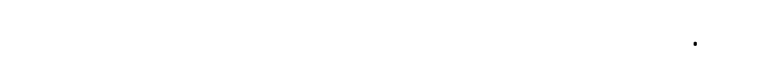

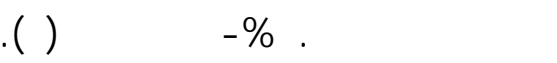

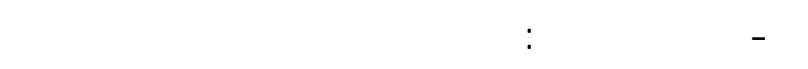

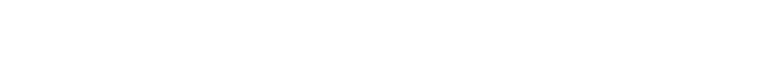

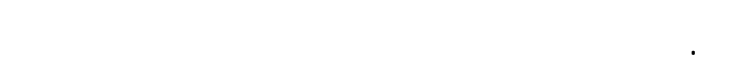
س

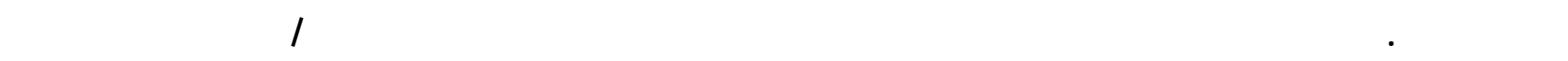

\begin{tabular}{|c|c|c|c|c|c|}
\hline لبنلن & "ألمانيا & اليونلن & روسيا & إلماليا & اللسنة / الولة \\
\hline 100.77 & IAн.r. & $10 \mathrm{~V} .7$ & $\therefore$ & $19 \% .74$ & 1991 \\
\hline $11 \% .7$. & $1 \wedge 0 . \mu q$ & 177.9. & $\therefore \cdots$ & $19 . .99$ & 1999 \\
\hline IEY.१V & IV१.४ & $19 \% . \Lambda$. & 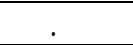 & $10 \varepsilon . \varepsilon r$ & $r \cdots$ \\
\hline $1 \mu 0 . \mu 0$ & $107 . \mu \wedge$ & 100.1. & $\therefore \cdots$ & IOr.IV & $r \cdots 1$ \\
\hline IEY.A. & $|\Lambda \cdot . r|$ & $r .1 .70$ & IVE.E. & 111.14 & $r \cdot r$ \\
\hline $1 \cdots .07$ & $101 . \mu^{\mu}$ & $10 \mathrm{~V} .1$. & $17 \varepsilon .77$ & $177 . \mu$ & $r \cdots \mu$ \\
\hline I Tr. & $179.9 \%$ & $1 \wedge 7.9 \mu$ & $1 \wedge 7.11$ & IVE.I. & $r \cdots \varepsilon$ \\
\hline $14 \cdot .91$ & $r 1 \cdot .71$ & 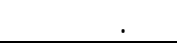 & $1 \Lambda \cdot . r \varepsilon$ & $r . \mu .77$ & $r \cdots 0$ \\
\hline 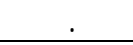 & IVH.ru & IVV.10 & $1 \Lambda \Lambda .7 V$ & IVV.9. & $r \cdots 7$ \\
\hline r.9.11 & ron.ru & rیר.r. & rru.rV & r१८.ह१ & $r \cdots V$ \\
\hline$\mu 79.9 \varepsilon$ & $\varepsilon 0 \wedge . \Lambda$. & $\varepsilon \vee \Lambda . \cdot V$ & r & 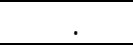 & $r \cdots \Lambda$ \\
\hline 7VE. $7 \varepsilon$ & 7Vץ... & 7Vर... & 7Vץ... & TVE. ${ }^{\mu} \varepsilon$ & $r \cdots q$ \\
\hline ror.r. & $010 . \cdot \mu$ & દน૫.દq & קו1. & $\varepsilon 7^{\mu} .99$ & $r \cdot 1$. \\
\hline દદץ.^૧ & нүب.00 & EVI.EV & $10 . .19$ & orl.9V & $r \cdot 11$ \\
\hline rVE.ro & orl.rq & $\varepsilon 7 \Gamma . \Lambda$. & & 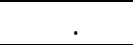 & $r \cdot 1 r$ \\
\hline гЕО.VV & r91.91 & rq7. 7 & 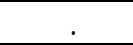 & r91.19 & المتوuط \\
\hline $1 \cdot .9$ & १.ह & $\mathbf{9 . 1}$ & - & $\mathbf{9 . \Lambda}$ & معل النمو \%(1) \\
\hline & & \multicolumn{2}{|c|}{ **2*منوي عند المستوى الاحتمالي 0.01} & \multicolumn{2}{|c|}{ *aعنوي عند المستوى الاحتمالي 0.05} \\
\hline
\end{tabular}




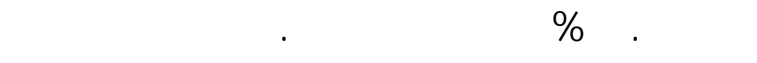

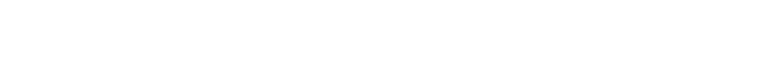

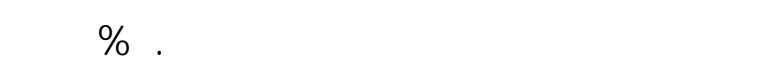

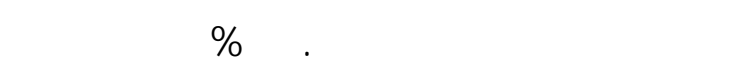

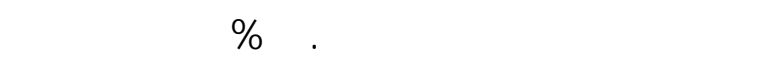
(1991 ץ - للسوق اليوناني: تراوح معلمل عدم الاسققرار للكمي ة المصدرة للسوق اليوناني بين حد أنف بلغ نحو 9 ـ . . \%

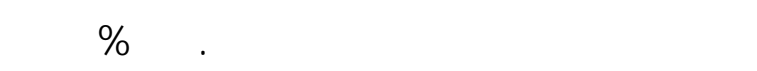

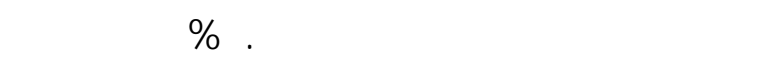

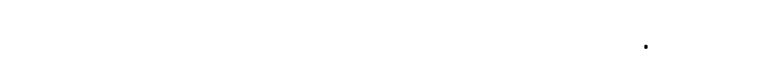

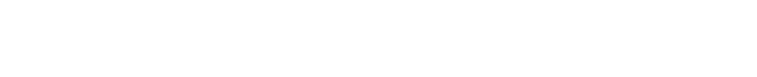

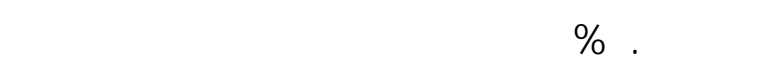
7

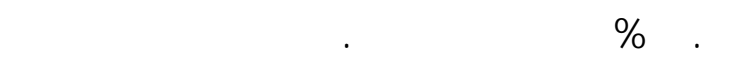

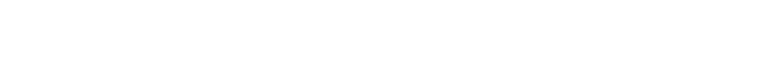

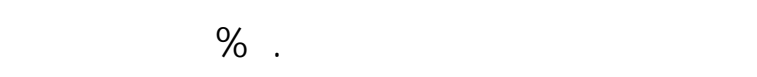

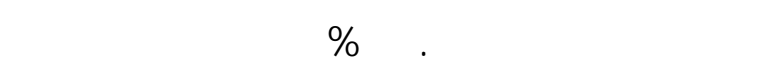

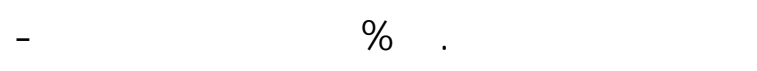

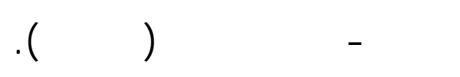
ع - للسوق الألماني: تراوح معلمل عهم الاستقرار للكمي ـة

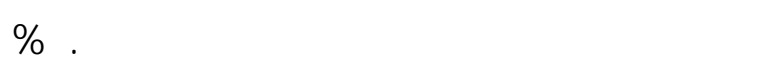

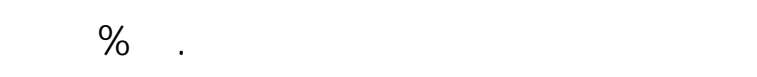

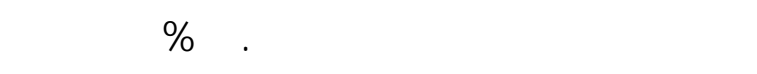

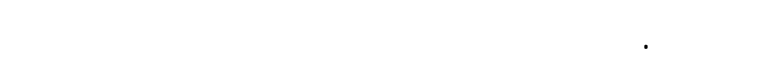

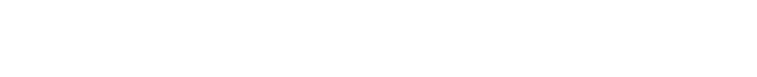

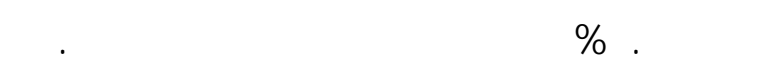

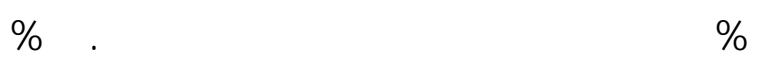

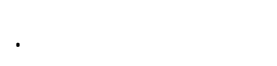

0 - السوق اللبنافي: تراوح عت لب عارط ـن الط لطس

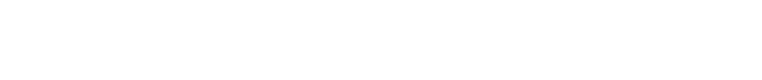

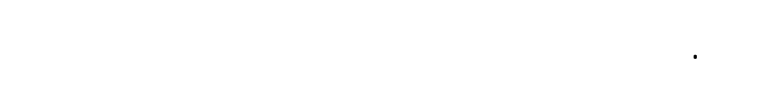

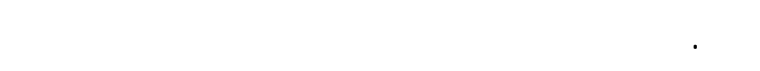

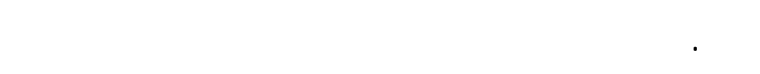
سنوي بلغ نحو 9 . - ا\% - جدول رقم (7) .

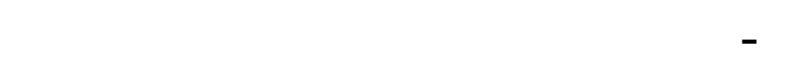 لأهم وأعلل الأسواق العالمية:}

I - للسوق الإيطالي: تراوح معلل عدم الانقرار للكمي ـة

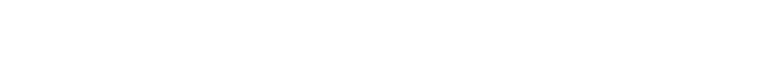

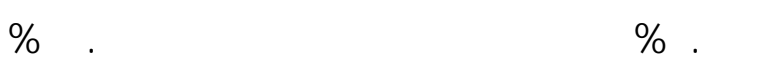

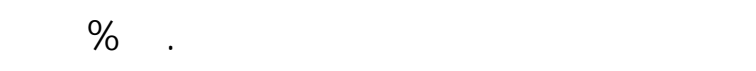

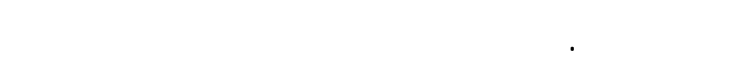

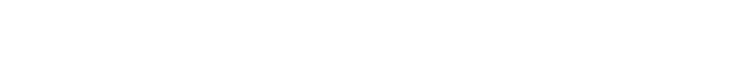

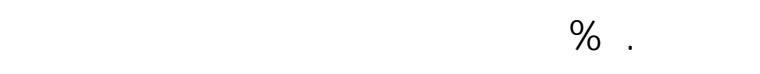

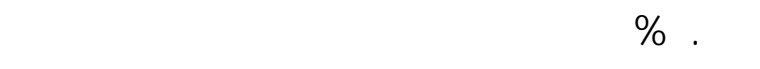

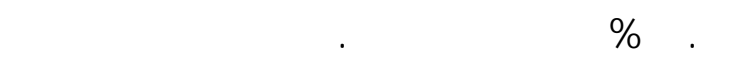

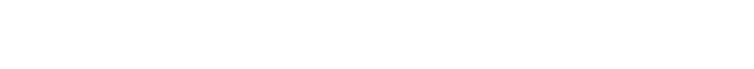

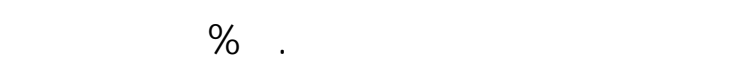

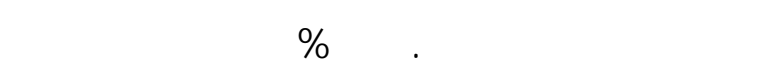

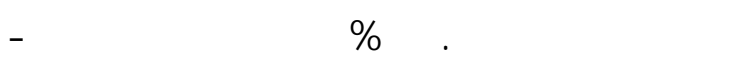

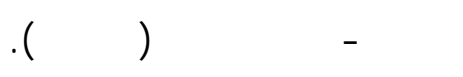
r - السوق الروسي: تراوح معلمل عدم الإنقرار للكمي ـة

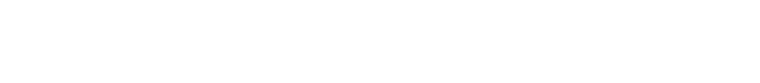

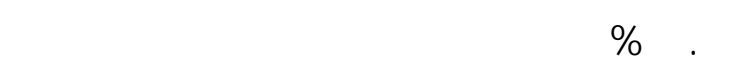
و

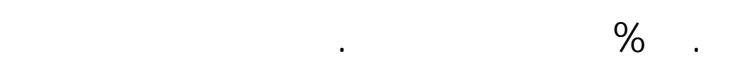

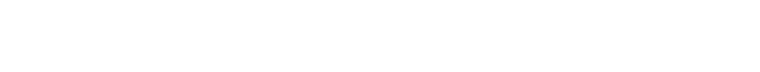

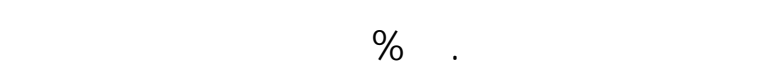

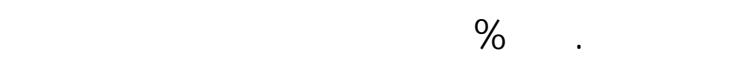




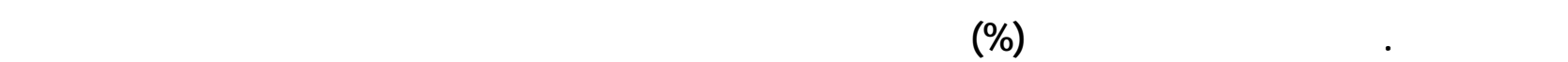

\begin{tabular}{|c|c|c|c|c|c|}
\hline & & & & \multicolumn{2}{|c|}{ 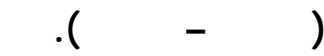 } \\
\hline لبنل & ألمانيا & اليونلن & روسيا & إيلاليا & المولة / المنة \\
\hline IV.r & $T \cdot . \varepsilon$. & $\therefore .9$ & $1 \cdots, \cdot$ & $07.0 \mathrm{~V}$ & 1991 \\
\hline$r \varepsilon_{.} \cdot \Lambda$ & ro.Ar & |r.MI & $1 \cdots, \cdots$ & ro.vr & 1999 \\
\hline 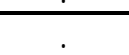 & $\varepsilon \varepsilon .{ }^{\varepsilon \mu}$ & 79.41 & $1 \cdots, \cdots$ & $19.1 \%$ & $r \cdots$ \\
\hline r.V7 & IE.भV & Vo.VV & $1 \cdots \ldots$ & 1.07 & $r \cdot \cdot 1$ \\
\hline $11 . \mathrm{V}$ & $11 . \varepsilon \Lambda$ & $\cdot . r \varepsilon$ & $\Lambda 9 . \mathrm{V}$ & V.r & $r \cdot \cdot r$ \\
\hline$\mu \cdot . \mathrm{VY}$ & 0.97 & $\varepsilon .07$ & rr.99 & HY.AV & $r \cdot \mu^{\mu}$ \\
\hline ro.e. & rA.VI & $11 Y .11$ & ET.ME & $1 \cdot . \Lambda \Lambda$ & $r \cdot \cdot \varepsilon$ \\
\hline 00.40 & $7 \varepsilon .7$. & $0 \% .7 \Lambda$ & $\varepsilon \varepsilon^{\mu} .7 \varepsilon$ & 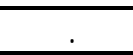 & $r \cdot .0$ \\
\hline $0 . . \mathrm{KA}$ & EY.TY & $7 \cdot . \mu \Lambda$ & $1 \Lambda . V^{\mu}$ & $7 \Psi . v \wedge$ & $r \cdot .7$ \\
\hline $1 \cdot \varepsilon_{\cdot} \cdot \mu$ & $\Lambda .09$ & r.Ar & 10.60 & rา. & $r \cdot \cdot v$ \\
\hline ro.Er & $77 . r \Lambda$ & IV. .0 & $1 . .1$ & $\varepsilon \cdot . \vee q$ & $r \cdot \Lambda_{1}$ \\
\hline$\% 9.70$ & E.VI & TY.MN & $79.7 \%$ & $0 \varepsilon . \cdot 1$ & $r \cdot . q$ \\
\hline r7.rq & $1 V .79$ & $\cdot . \mathrm{M \Lambda}$ & $\mu \varepsilon . V Y$ & 11.7 & $r .1$. \\
\hline $00 \cdot 1$ & $7 . r 1$ & E.rE & IVT.91 & 0.10 & $r .11$ \\
\hline IN.VT & \%9.9V & \%q.Vo & YV.A & $\varepsilon 0.10$ & $r \cdot I r$ \\
\hline$r 7.07$ & 11.7. & 1.70 & 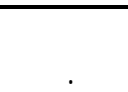 & $r \cdot r o$ & المتهنوس \\
\hline
\end{tabular}

المصدر: جمعت ومسبت من بيانت جدول رقم (r).

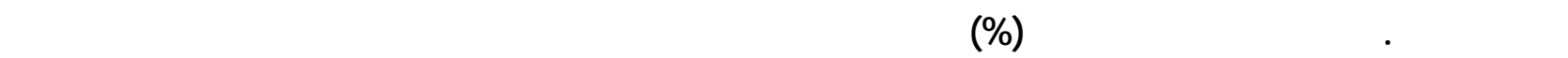

\begin{tabular}{|c|c|c|c|c|c|}
\hline لبنلن & ألمانيا & اليونلن & روسيا & إلماليا & لللسنة / الدولة \\
\hline$\Lambda १ . \wedge \varepsilon$ & $r \cdot .0 \mathrm{~V}$ & $00 . \Lambda \Lambda$ & $1 \cdots$ & EV. $\left.{ }^{\mu}\right\rceil$ & 1991 \\
\hline $0 . \cdot V$ & 07.49 & $11.7 \mu$ & $1 \cdots$ & भА. ११ & 1999 \\
\hline $7 . V^{\mu}$ & щq.ह0 & $7 r .91$ & $1 \cdots \ldots$ & 14.17 & $r \cdots$ \\
\hline $0 . r o$ & rE.AV & $\Lambda \cdot . \cdot 7$ & $1 \cdots \cdots$ & $1 \mu . V^{\mu}$ & $r \cdots 1$ \\
\hline rI.VE & $1 \Lambda . \cdot 1$ & $7 . r \varepsilon$ & 9 9Y.Oץ & rI.r. & $r \cdot r$ \\
\hline$r \wedge .90$ & re.01 & $\mu \mu^{\mu} \cdot \mu$ & $0 \Lambda . \mu 1$ & $9.7 \varepsilon$ & $r \cdots \mu$ \\
\hline 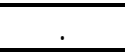 & $0.0 \varepsilon$ & $\varepsilon \wedge .77$ & $7 V . \varepsilon I$ & rq.ro & $r \cdots \varepsilon$ \\
\hline 19.07 & YV.YY & ro. & $V \cdot .01$ & $9 . \varepsilon 0$ & $r \cdot 0$ \\
\hline Vo.ro & $1 \cdot .70$ & $9.7 \varepsilon$ & 07.71 & $1 \cdot .1$. & $r \cdots \tau$ \\
\hline IrY.9r & r1.09 & IV.EV & EV. 7V & $\Lambda .9 \mu$ & $r \cdot v$ \\
\hline 0.09 & OH. E & 00.90 & $|\cdot . \mu|$ & 10.99 & $r \cdots \Lambda$ \\
\hline rY.I. & $90 . r 0$ & rV.हq & $\varepsilon \varepsilon . \mu^{\mu}$ & $r \cdot . I V$ & $r \cdots q$ \\
\hline rV.0. & $7 . \wedge \mathrm{V}$ & $\Lambda . V Y$ & r0.9ह & $\cdot . \mathrm{VI}$ & $r \cdot 1$. \\
\hline$\varepsilon \wedge . Y \mid$ & $11 . \varepsilon \mu$ & V.हV & |OV.r| & $10 . V 1$ & $r \cdot 11$ \\
\hline ro.99 & 01.71 & rา.0r & 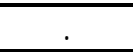 & $\varepsilon 7 . \cdot 7$ & $r \cdot 1 r$ \\
\hline r...7 & rн.90 & re.01 & $71.0 \varepsilon$ & $17 . r 1$ & المتوبط الهiهيوي \\
\hline
\end{tabular}

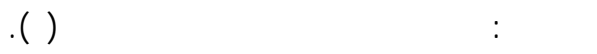


لحمد توفيق الفل ولخرون.،: تحلل اقتصادي قيلسي لمحددات الكفاءة التسويقية ومحددات صادرات الططلس المصرية

جطل رقم 9.

(r.) - 1994)

\begin{tabular}{|c|c|c|c|c|c|}
\hline لبنلن & ألمانيا & اليونلن & روسيا & إلماليا & للمنة / الدولة \\
\hline$r \cdot 9 . \wedge \varepsilon$ & IIr.V. & V9.1Y & $1 \cdots$ & Iro.v7 & 1991 \\
\hline$\varepsilon 0 . \mu r$ & $7 \cdot . \varepsilon 7$ & $\varepsilon r .1$. & $1 \cdots \ldots$ & $7 \mu . V r$ & 1999 \\
\hline 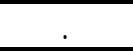 & rщ.V7 & $\mu . .97$ & $1 \cdots, \ldots$ & $0 . \varepsilon \varepsilon$ & $r \ldots$ \\
\hline$\cdot .94$ & $|\cdot . \mu|$ & $I r . \mu V$ & $1 \cdots \ldots$ & $1 \mu .9 \mu$ & $r \cdots 1$ \\
\hline $11 . \wedge \varepsilon$ & 11.00 & r.EV & $0 \Lambda .7 V$ & 14.07 & $r \cdot r$ \\
\hline EV. . 0 & ro.1ع & r".01 & $14 . \mu 9$ & $\mu . .9$ & $r \cdot \mu$ \\
\hline है. ११ & ro.rर & rq.A. & r.عr & ro... & $r \cdots \varepsilon$ \\
\hline $0 . .11$ & rV.AV & $19.0 \%$ & 17.0 & rl.V. & $r \cdots 0$ \\
\hline Or.VV & $\varepsilon 7.1$. & $\varepsilon 0 . \tau^{\mu}$ & 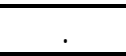 & $\varepsilon 0.10$ & $r \cdots r$ \\
\hline r.o. & rา. & $19.0 \mathrm{r}$ & rr. 7 & $17 . \Lambda \mu$ & $r \cdots v$ \\
\hline Ir.ro & $r \cdot .7 \Lambda$ & $r \varepsilon .0$ & Ir.V & ro. 7 & $r \cdots \Lambda$ \\
\hline$\Lambda \Lambda . V \cdot$ & 7ह."I & $7 r .11$ & $\Lambda \Lambda . \varepsilon \Lambda$ & $7 \cdot . V Y$ & $r \cdots q$ \\
\hline$\Lambda .7$. & IV.Tr & 1.19 & V.Eา & $\mu .1 \mu$ & $r \cdot 1$. \\
\hline V.rA & rr.rq & $\cdot .74$ & 18.97 & $\Lambda .7 \Lambda$ & $r \cdot 11$ \\
\hline $10.1 \mathrm{~V}$ & $\varepsilon . V Y$ & $\Lambda . r 7$ & $r \cdot .1$. & $1 \varepsilon .91$ & $r \cdot 1 r$ \\
\hline$r \cdot V r$ & r7.rA & 10.v. & rA.V. & rI.AN & المتوبط الهiهuي \\
\hline
\end{tabular}

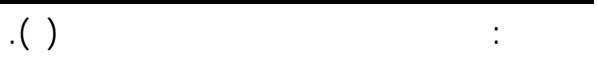

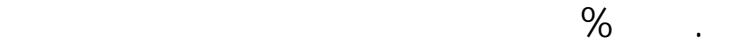

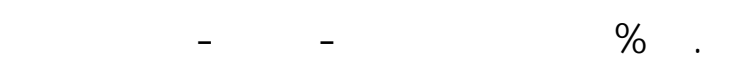
.$(\Lambda, 9, \vee)$

- 1 - الواردت العالميةمن البملس لأهم وأعلل الأسوقة

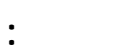

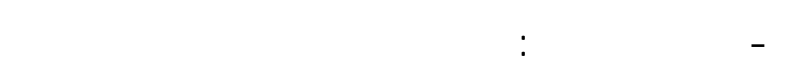

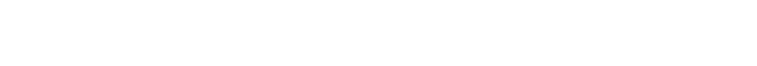

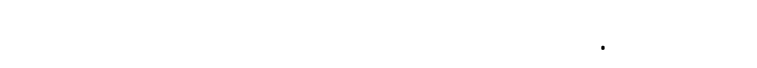

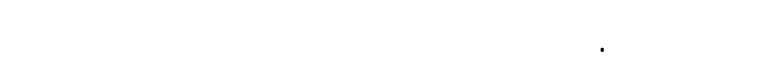
بلغ حوالي عَr.

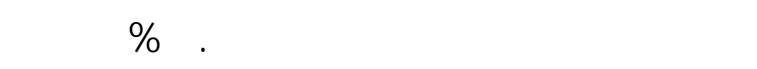

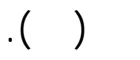

r - للسوق الروسي: تب ــن أن الـ ـواردات العالمي ـة مـ ـن

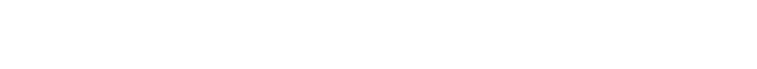

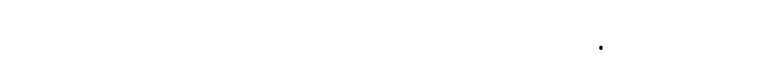

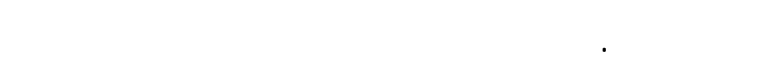

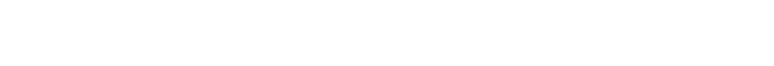

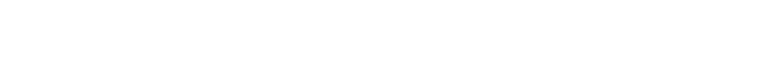

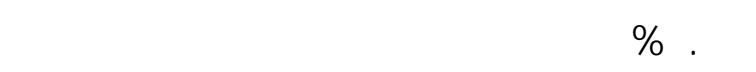
و 1994 ، بمتوسط هidir.V. ^r.r\%

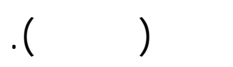
0 - للسوق اللبناني: تراوح معلل عهم اللس تقرار للكمي ـة

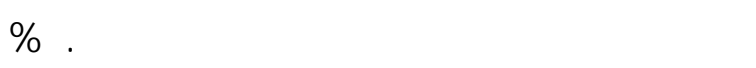

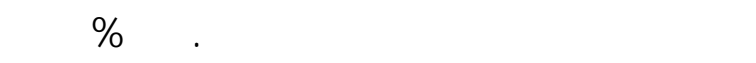

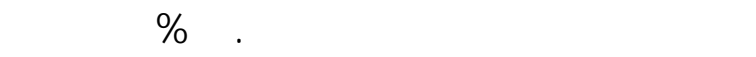

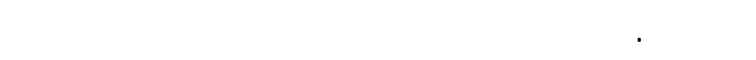
صادرات الططس اللسوق اللبنافي بين حد أنف بلغ نحو

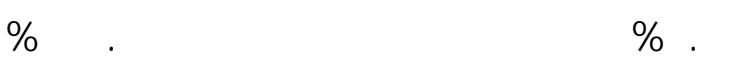

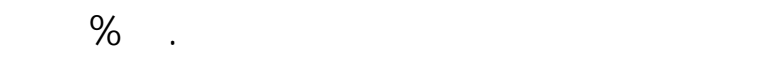
فترة الدرلسة. فى حن تراوح معلمل ع دم الآس نقرار

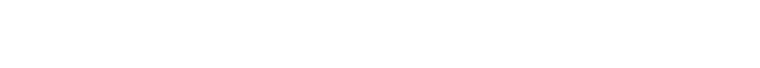

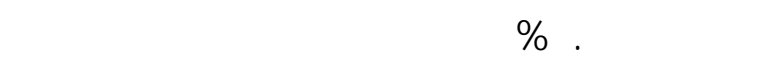




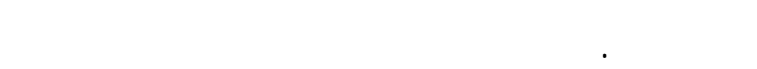

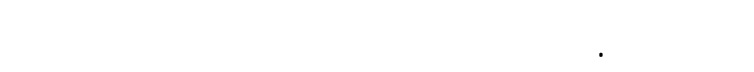

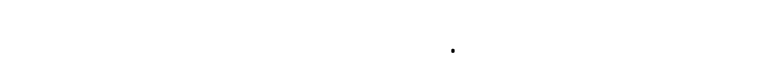

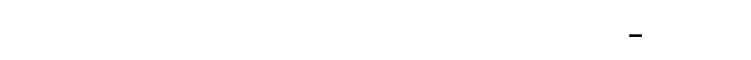
- جدول رقم ( - ().

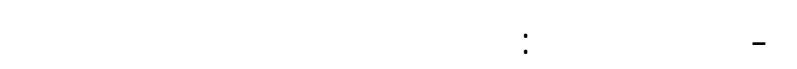

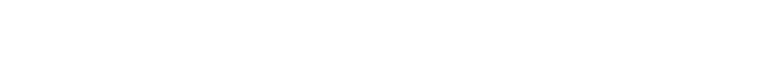

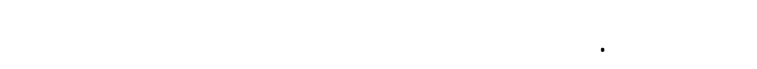

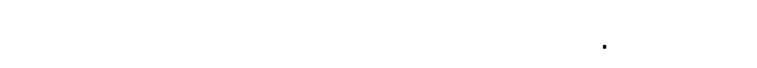

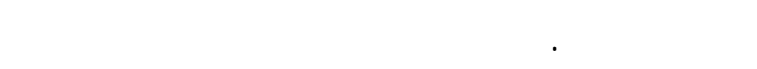

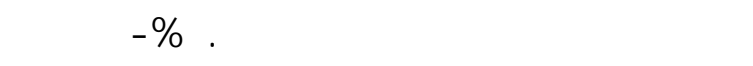

$$
\text { رقم ( ) (1). }
$$

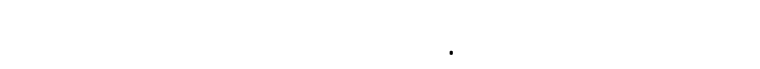

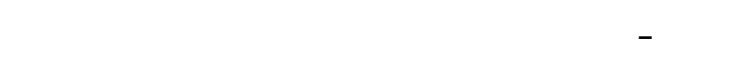

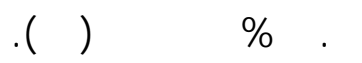

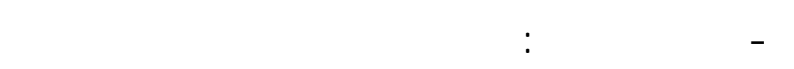

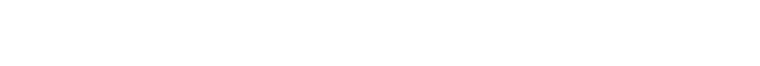

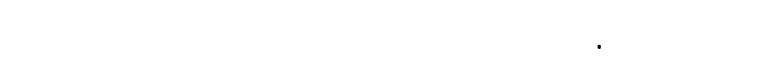

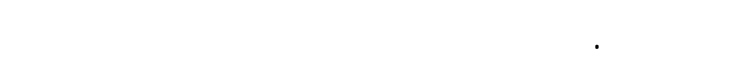

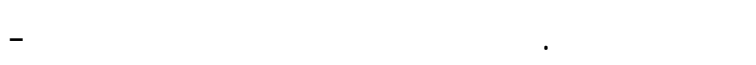

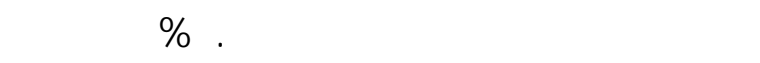

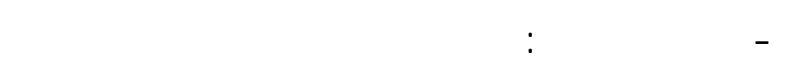

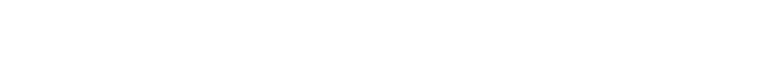

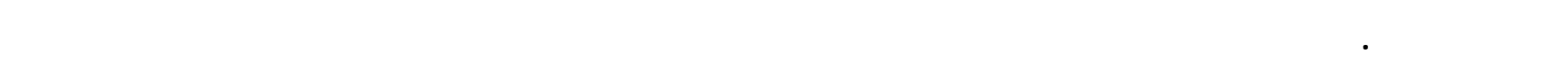

\begin{tabular}{|c|c|c|c|c|c|}
\hline & & & & \multicolumn{2}{|c|}{ الفترة (1994 - או -r). } \\
\hline ل البنلن & ألملنيا & اليونل & | روسيا & إيلاليا & |للسنة / الولة \\
\hline$\varepsilon 0 . \mu_{1}$ & 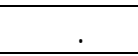 & $\mid$ |Yץ.हा & Iस & $\varepsilon 01.17$ & 1991 \\
\hline$\varepsilon 7 . \wedge \varepsilon$ & $7 \varepsilon \Lambda .91$ & $1 \mu \mu . V \mu$ & $r \cdot \varepsilon . r \Lambda$ & 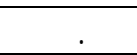 & 1999 \\
\hline EE. ${ }^{\mu}$ & 0.1 .11 & I.E.00 & rา१.广^ & $01 \cdot .7 \mu$ & $r \ldots$ \\
\hline $0 \varepsilon . \cdot \Lambda$ & $0 \wedge 9.11$ & $\mathrm{VY} . \mathrm{V} 7$ & $1 \cdots .1 \mathrm{~V}$ & 011.11 & $r \cdot .1$ \\
\hline$V \varepsilon . \wedge V$ & OVV.11 & IIE. EO & 194.99 & $001.7 \pi$ & $r \cdot \cdot r$ \\
\hline $\mathrm{V} 7.09$ & 009.09 & 100.11 & $0 \cdot \Lambda .1$ & $7 \pi r .90$ & $r \cdot \mu^{\mu}$ \\
\hline 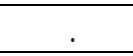 & $O \mu_{\mu} \Lambda \cdot r$ & IEI.rך & $171 . r r$ & $74 \cdot .97$ & $r \cdot \cdot \varepsilon$ \\
\hline$\Lambda \Lambda .7 \pi$ & $091.9 \mathrm{~V}$ & $11 V . V \varepsilon$ & rV7.01 & OVץ. ${ }^{\top} 7$ & $r \cdot .0$ \\
\hline VO.VE & $7 V V . \cdot r$ & $14 \cdot .9$ & $\mu V \cdot .9 \mu$ & $0 \% 9.77$ & $r \cdot \cdot 7$ \\
\hline $11 V .70$ & 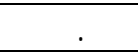 & $1 \varepsilon \cdot .9 \pi$ & rol.V & OVT. ${ }^{\mu \mu}$ & $r \cdot \cdot v$ \\
\hline 9r.rk & OPr.Eस & 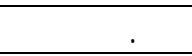 & OHV.દq & 099.97 & $r \cdot \Lambda_{1}$ \\
\hline Ar.rV & OVA.YE & 170.17 & FVE. 10 & 7.r.ro & $r \cdot q$ \\
\hline IIr.r. & OVH.V7 & $1 \mu^{\mu} . \varepsilon \mu$ & $77 \mathcal{T} .{ }^{\mu} \mathrm{r}$ & $V \cdot \Lambda . V \varepsilon$ & r.l. \\
\hline $99 . \wedge \mu$ & 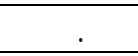 & IVE.rq & IE77. Y & $7 \cdot r \cdot \Lambda$ & $r .11$ \\
\hline $79.0 \mu$ & VEา. $\cdot \varepsilon$ & $1 \mathrm{Ir} 9.01$ & $\varepsilon 71.1 \mathrm{r}$ & $77 V . \cdot r$ & $r \cdot 1 r$ \\
\hline VV.rE & $7 \cdot 7.11$ & IYr.1. & $\varepsilon \cdot 0.1 r$ & OVT.re & المتوطy \\
\hline \multirow[t]{2}{*}{${ }^{* *} 0.0$} & - & ${ }^{*} r . \varepsilon$ & ${ }^{* *} 11.7$ & ${ }^{* *} \mathrm{r.0}$ & معدل النمو(1) \% \\
\hline & & الحتمالي 0.01 & 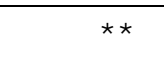 & 0.05 & ندوي عند المستوى الا \\
\hline
\end{tabular}




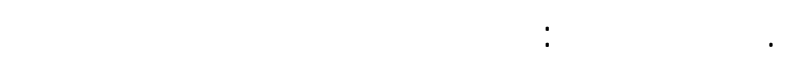

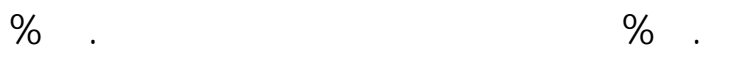

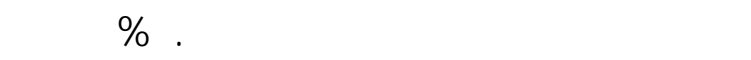

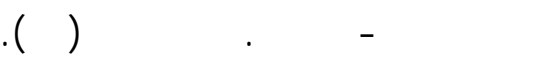

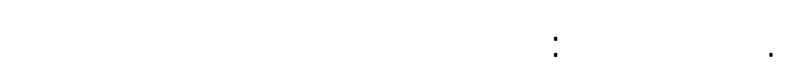

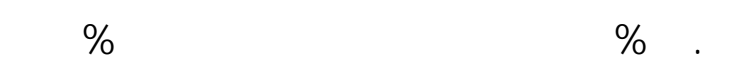

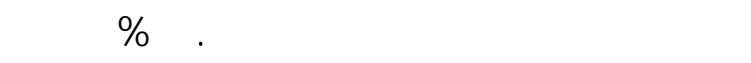

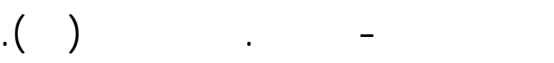

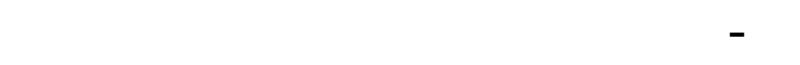
العالمية: بليستعراض بيبانت جدول رقم( (I) يتضح عنم وجود

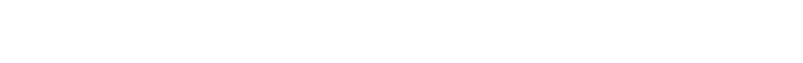

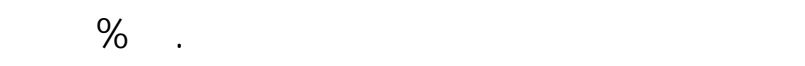

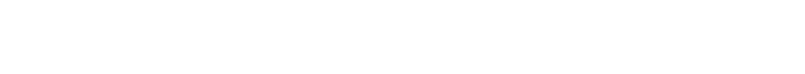

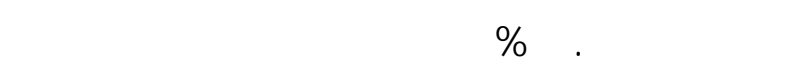
r. r. 11 \% $\%$ V.r.r
1 I -مؤشر النصيب اللسقي: بلسيتعراض مؤثر النصيب

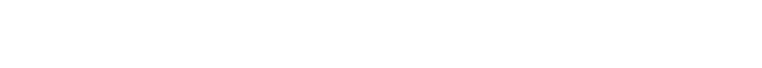

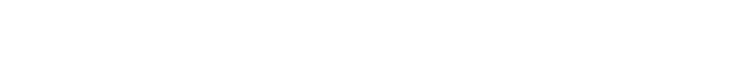
مايلي: -

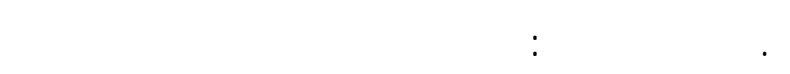

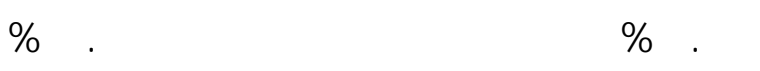

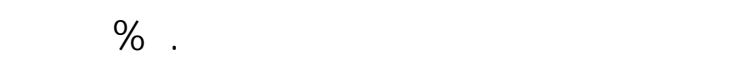

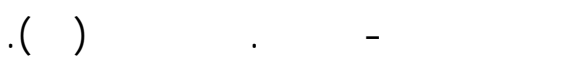

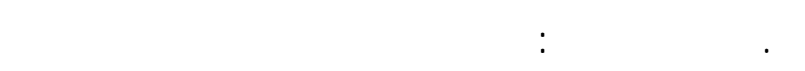

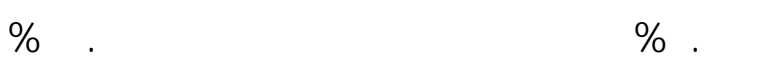

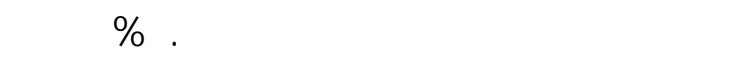

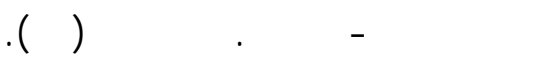

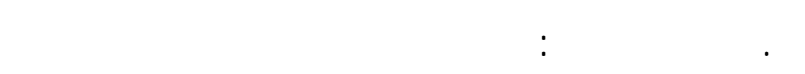

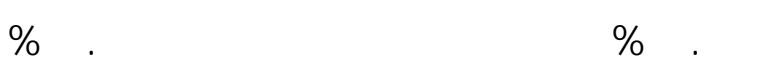

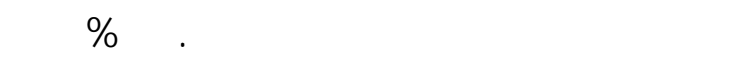

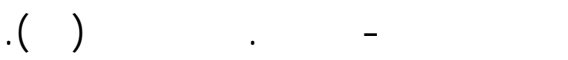

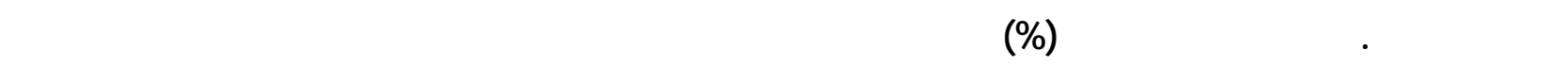

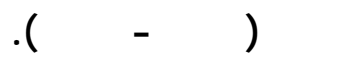

\begin{tabular}{|c|c|c|c|c|c|}
\hline لبنلن & ألملنيا & اليونل & روسيا & إلياليا & اللسنة / الدولة \\
\hline$V T . \cdot 1$ & $V . r$ & 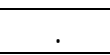 & & $\varepsilon \cdot \cdot V$ & 1991 \\
\hline $7 \% .01$ & 11.07 & $\mu . V^{\mu}$ & $\therefore$ & V.7. & 1999 \\
\hline Vo... & $7 .{ }^{\mu}$ & $1 \varepsilon .0 \mu$ & $\because \ldots$ & V.r $\mu$ & $r \cdots$ \\
\hline $79.1 \%$ & $\Lambda \cdot \cdot \varepsilon$ & $17.0 \%$ & $\because$. & $9.7 \mathrm{~V}$ & $r \cdot .1$ \\
\hline$\varepsilon 0.7$. & A. 10 & દ..१1 &.$\Lambda \varepsilon$ & 1.00 & $r \cdot r$ \\
\hline 70.77 & 9.70 & ro. $\varepsilon$ & $\varepsilon .11$ & $\left|1 . \mu^{\prime}\right|$ & $r \cdot \mu^{\mu}$ \\
\hline 71.99 & $11.7 \mathrm{~V}$ & V१.१५ & IY.VN & 9.79 & $r \cdot \varepsilon \varepsilon$ \\
\hline 77.VV & $|r . \Lambda|$ & $v \cdot . v$. & V.EV & I\%.\%. & $r \cdot .0$ \\
\hline 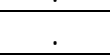 & १.ץ & $7 V .00$ & $1 \% . \varepsilon \varepsilon$ & $1 \Lambda . \cdot \varepsilon$ & $r \cdot 7$ \\
\hline $70 . \varepsilon$. & V.r $\mu^{\mu}$ & " $\Lambda .79$ & 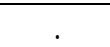 & $1 \% .0 \%$ & $r \cdot v$ \\
\hline $0 \cdot .99$ & r.oE & $\varepsilon 7.9 \mu$ & $1 \varepsilon .9$ & $1 \varepsilon .90$ & $r \cdot \Lambda$ \\
\hline TV.YN & $7 . \wedge \mathrm{V}$ & YY.YV & V.V7 & $0 . r$ & $r \cdot . q$ \\
\hline$r \cdot .9$. & $0.1 \mu$ & $\varepsilon \varepsilon . \cdot 9$ & $1 . .01$ & $\Lambda . \varepsilon \Lambda$ & $r \cdot 1$. \\
\hline $17.7 \mu$ & $0 . \varepsilon \mu$ & rr.qr & $r \mathrm{r} \cdot \mu^{\mu}$ & $1 . .9 \Lambda$ & $r .11$ \\
\hline ET.qV & 0.9 . & YN. ${ }^{\top}$ & IV.0. & $0.9 \varepsilon$ & $r \cdot I r$ \\
\hline$\varepsilon 7 . \varepsilon 1$ & V.rY & YV.IV & 9.7 & 9.11 & المتوبط الهنهسي \\
\hline
\end{tabular}




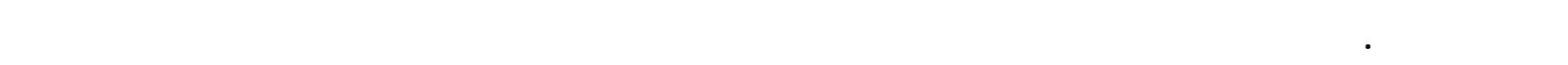

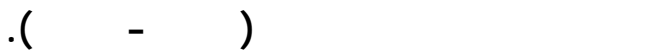

\begin{tabular}{|c|c|c|c|c|c|c|c|}
\hline معللى التركز الجغرلي \% & دل لخرى & لبنلن & ألمانيا & اليونلن - اليون & תפטيا & ليلاليا & للسنة / الدولة \\
\hline$\varepsilon \Lambda . \mathrm{O \mu}^{\mu} \mathrm{r}$ & $\cdot .1 \cdot \mathrm{V}$ & $\therefore \cdot r$. & $\therefore .09$ & $\cdot . \varepsilon \mu$ & $\ldots \cdots$ & $\therefore \cdots 7$ & 1991 \\
\hline$\varepsilon \Lambda . \cdot \varepsilon \varepsilon$ & $\because \cdot \Lambda \mathrm{V}$ & $\because \cdot 1 \varepsilon$ & $\therefore \wedge \mathrm{T}$ & $\therefore \cdot r \Lambda$ & $\therefore \cdots$ & $\cdot . .1 \mathrm{~V}$ & 1999 \\
\hline ع7. & $\therefore \cdot \pi$ & $\therefore$. 10 & $\cdot . \cdot \varepsilon l$ & $\therefore \cdots 9$ & $\therefore \cdots$ & $\therefore .01$ & $r \ldots$ \\
\hline$\varepsilon V .0 \cdot \varepsilon$ & $\cdot . \varepsilon \varepsilon$ & $\therefore \cdot \varepsilon l$ & $\therefore 70$ & $\therefore \cdots \varepsilon$ & $\therefore \cdots$ & $\therefore \cdot \mathrm{VI}$ & $r \cdot 1$ \\
\hline 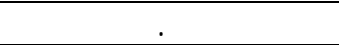 & $\cdot . \cdot \varepsilon \varepsilon$ & 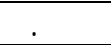 & $\cdot . \varepsilon r$ & $\therefore .0$. & $\ldots \cdots$ & $\cdot . \cdot \varepsilon r$ & $r \cdots r$ \\
\hline Er.7Tr & $\cdot . r \varepsilon$ &..$r q$ & $\therefore \mu \mu$ & $\cdot . \mu \varepsilon$ & 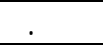 &. $.0 \mathrm{~V}$ & $r \cdots \mu$ \\
\hline 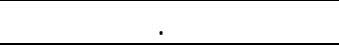 & $\therefore \cdot \mu \Lambda$ &. .17 & $\therefore \cdot Y V$ & $\because \cdot \Lambda \Lambda$ & $\therefore \cdots \mu$ & $\cdot . r 7$ & $r \cdots \varepsilon$ \\
\hline 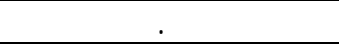 & $\therefore$. १q & $\therefore \Gamma^{\mu}$ & $\therefore . \mu q$ & $\therefore \varepsilon 0$ & $\therefore \cdots$ & $\therefore \cdot \mu \Lambda$ & $r \cdots 0$ \\
\hline દદ.દ૦V & $\therefore \cdot 1 \mathrm{~V}$ &.$\ldots \mu$ & $\therefore \cdot \mu$. & $\therefore .01$ &. .19 & $\therefore \cdot \mathrm{VI}$ & $r \cdots \tau$ \\
\hline$\varepsilon \mid . \Lambda \cdot \varepsilon$ & $\therefore . \mu 7$ & $\therefore \cdot \varepsilon$. & $\therefore \cdot 1$. & $\therefore \cdot r$. & $\therefore . r 7$ & $\cdot . \varepsilon r$ & $r \cdot V^{2}$ \\
\hline$\varepsilon \varepsilon . \cdot \Lambda 9$ & $\therefore \varepsilon 0$ &. .17 &..$\cdots 1$ & 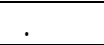 & $\cdot . \cdot \varepsilon r$ &. .01 & $r \cdots \Lambda$ \\
\hline EY. 170 &. .01 &. .11 & $\therefore \mu \varepsilon$ & . • $\varepsilon \varepsilon$ & $\therefore \cdot 1 \wedge$ & $\therefore \cdot r$. & $r \cdots q$ \\
\hline$\left.\varepsilon \mu^{\mu} . \mid \varepsilon\right\rceil$ & $\cdot . \mu \mathrm{V}$ &.$\cdots 7$ &. .1. & $\therefore . \mu q$ &. $.0 \varepsilon$ & $\cdot . \varepsilon$. & $r \cdot 1$. \\
\hline 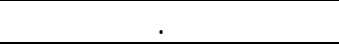 & $\therefore \varepsilon \bar{T}$ &.$\cdots 1$ & 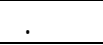 & $\because \cdots \Lambda$ &. .509 &. .11 & $r \cdot 11$ \\
\hline$\varepsilon \mu . \mu १$ & $\cdot$. •२ &. .1 . & $\cdot . r \mu$ & $\therefore \cdot 10$ & $\cdot . \mathrm{V} \varepsilon$ & $\cdot .11$ & $r \cdot i r$ \\
\hline EO. rOE & $\cdot . \varepsilon \Lambda$ & $\cdot . r$. & $\therefore \mu \mu$ & $\therefore \mu \mathrm{O}$ & $\cdot . \mu \varepsilon$ & $\therefore \mu \mu$ & متوuط الهنهي \\
\hline
\end{tabular}

المصدر: جمعت ومسبت من البيانت الواردة بالجدول رقم (؟).

- إجمالي الطاقة الإبستهلكية القومية من البططس + الأف

$$
\text { طن =س }
$$

- لأسعار التجزئة المحلية للططلس المصرية بالجنيه الطا ـن

$$
\text { . }
$$

- الأسعار التصديرية المصرية للططس بالدولار الطن =

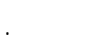

- متوسط السعر العالهي لصادرات الططسس بالدولار الطن

$$
.00=
$$

- النبة بين إجمالي الصادرات المصرية من الططس إلي لجمالي الناتج المحلي منها (مؤثر ق ـوة التص حير)

-سعر الصادرات الهولنية من الططس بالدولار الطن=

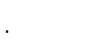

-سعر الصادرات الفرنسية من الططس بالدولار الطن =

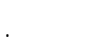
- كمية نقاوي الططس المستورة بالأف طن =سوه.
ثالثاً - محددت الالب الخارجي المؤثرة علمي صد الرات البمالمل المصربة لأهم وأعل الأسوق العالمية خلا

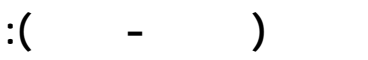
يهم البحث بصياغة الفروض البحثية للعلاقة للسبية بين

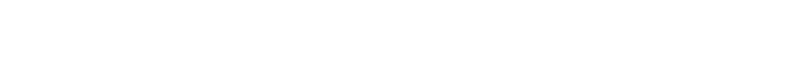
المصرية وبين المتغيرات الفسيرية، والتي على لسٔسها يقم

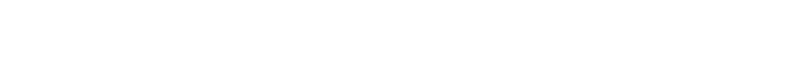

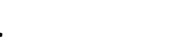

\section{الافترالك البحثية:} قم بناء أربعة نماذج قيلسية لستخلمت فيه Lا الأس عار، وهي النموذج الخطي والنم _وذج اللوغ ارتيهي والنم _وذج النصف لوغارتيهي في (ص) والنموذج النصف لوغارتيهي فـ (س) والنموذج اللوغارتيمي العكسي. المتغير التالع: صح إجمالي كمية الصادرات المصرية من البطلس بالاف طن فى اللسنة. المتغيرات المسقلة (الفسيربة): إجماله الناتج المحلي من البطس بالأف طن =س المغ 
لحمد توفيق الفل ولخرون.،: تحليل اقتصادي قيلسي لمحددات الكفاءة التسوقية ومحددات صادرات الططلس المصرية 
حيث كلما قلت قيمته كلن ذلك دليلا عل ق Error (RMSE)

أفضلية (جوة) النموذج المستخدم في التنبؤ، وقد تبر بن أن

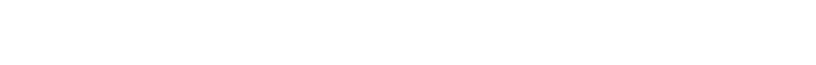

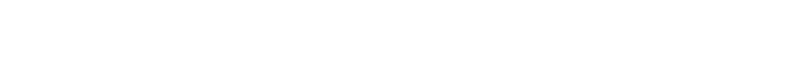

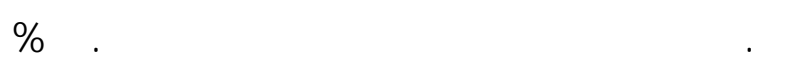

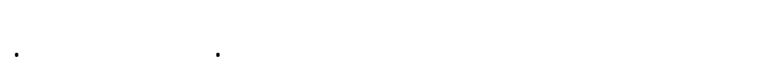

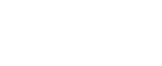

لحمد محمد توفيق الفيل (ولخرون)، أسلسيت علم الاقتصاد

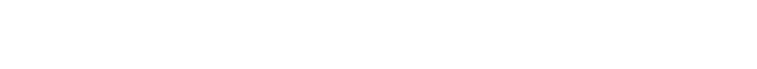

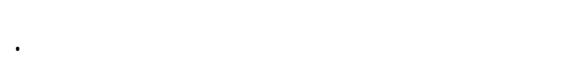
الجهاز المركزي للتعبئة العلمة والإحصاء، نش _رة لأس عار الجدار الجملة والتجزئة، القاهرة، أعداد مقفرقة. الجهاز المركزي للتعبئة العلمة والإحصاء، النثرة للس ـنوية للتجارة الخارجية، أعداد مختلفة. الجهاز المركزي للتعبئة العلمة والإحصاء قاء ــة بيان ـلت

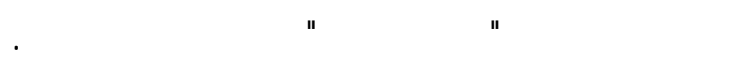

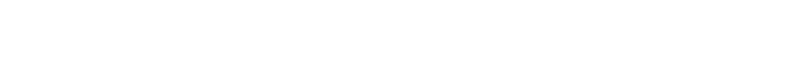
المصرية والمركز التنافسي لها، مجلة البحوث والتنمية

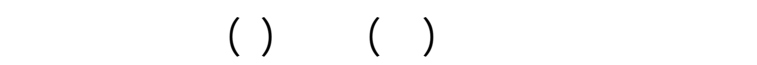

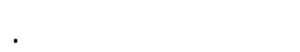

سلمح محمد هرن عليشهلب، درلسة إقتص الية تحليلي ة لأهه الصادرات الزراعية المصرية إلى الدول العربية،

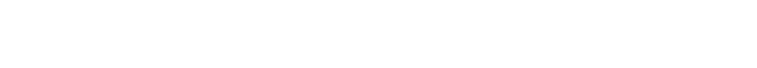

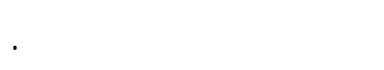

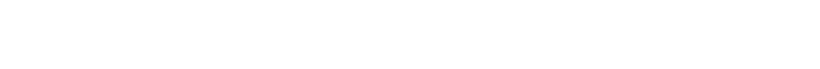

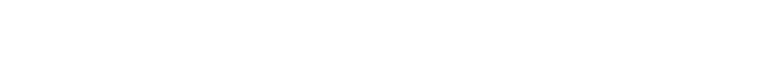

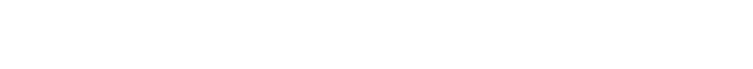

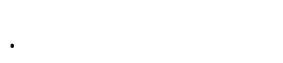

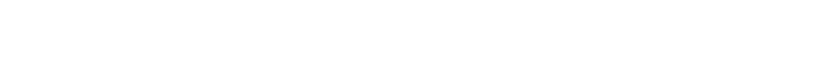

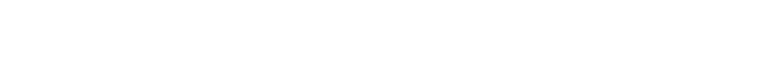

وتوضح المعادلة التالية نتائج القير القيلسي لدالة الالب عل صاررات البللاس المصربة حيث المكن الحصول على النتائج التالية:

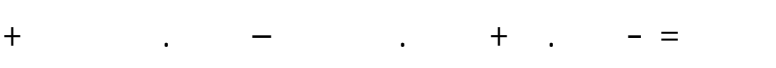

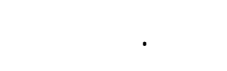

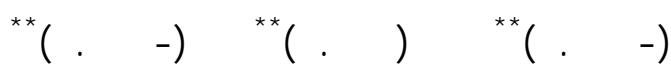

ر ر بدرلسة العلاقة بين كمية الص ادرات المص -رية م ن

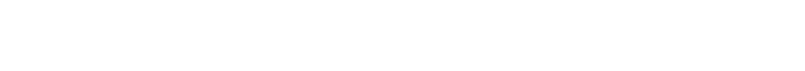

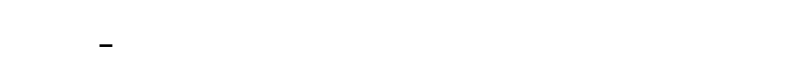
بلستخده الانحدار المتعدد للتعرف عل م أه م المتغي _رات

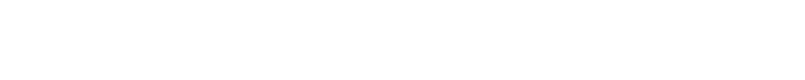

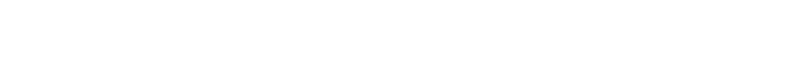

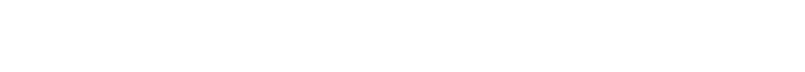

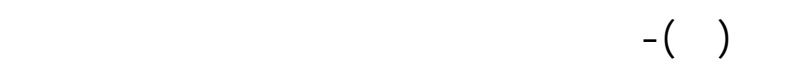
الصادرات المصرية من الططس ولجمالي الانتاج المحلي

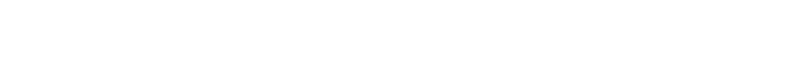

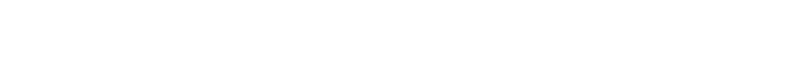

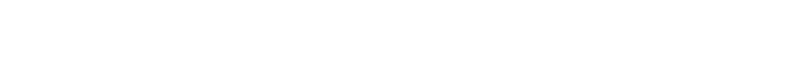

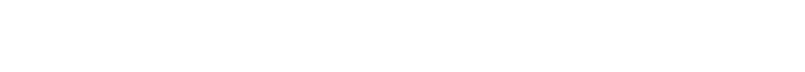

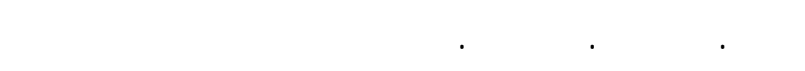
يعنى أن المتغيرات الفسيرية التي تتضمنها نماذج الانحدار

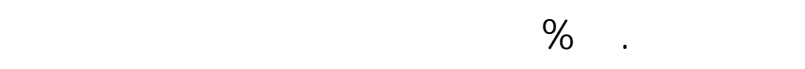
الترتيب. وهذا يعكس أنه كلما ارتفعت الطاقة الإس التيعابية كلما زادت الصادرات المصرية - جدول رقم (ب ا). وبدرلسة الظور المسقبلي كمية الصادرات المص -رية

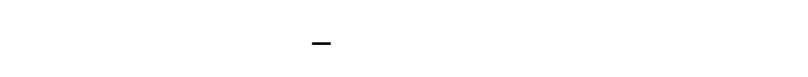
طريقة هولت ذات المعلمين وقد تمت المفاضلة بين نم اذج التنبؤ المختلفة لاختيار افضل نموذج للتنبؤ وذلك وفة لمعيار الجذر التربيعي لمتوسط مريع الظأ Root Mean Squared 
لحمد توفيق الفل ولخرون.،: تحلل اقتصادي قيلسي لمحددات الكفاعة التسويقية ومحددات صادرات الططس المصرية

التصدير الرئيسية في مصر، المجلة المصرية للاقتصاد منف لأحمدسليم ولخرون، درلسة القتصايلت إنتاج وتسويق

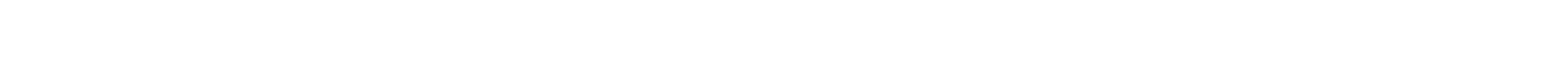

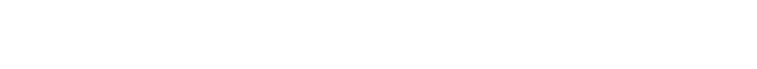
القاهرة. العدد الرالع، سيسمبر rا • r، القاهرة. صلاح محمود عبد الممسن عرفة، درلسة تحليلية للتج ارة وزارة الزراعة ولستصلاح الأراض مي، قط اع للث ـأون

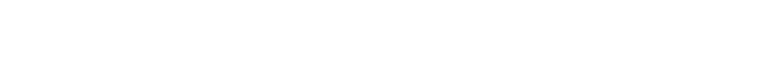

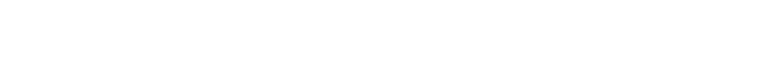

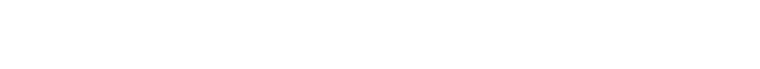
القاهرة.

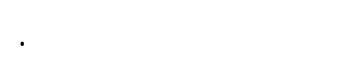
وزارة الزراعة ولستصلاح الأراض مي، قط اع للث ـأون

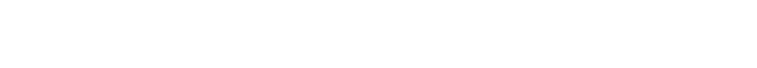
مصر العربية، أعدادمختلفة، الفاهرة. وزارة الزراعة ولستصلاح الأراض مي، قط اع للث ـأون

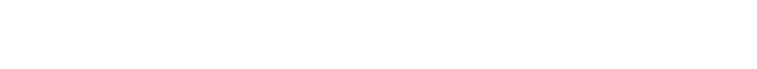
الاقتصاد الزراعي، أعداد مقرقة.

http:// www. Fao . org http://comtrade.un.org/db www.capmas.gov.eg. محمود عبد الهاديشافعي، محاض _رات ف مي الإقتص اد القيلسي المقنم، محاضرات لطلبة الدرلست العليا، قم

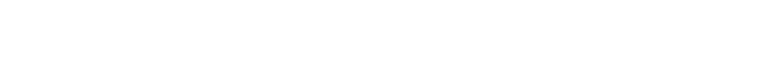
r.r. محمود عبد الهاديشافعي، محاضرات تحلي لل للسلمد لـ

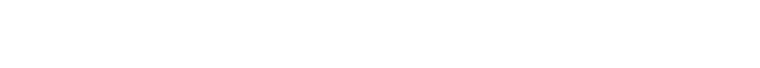

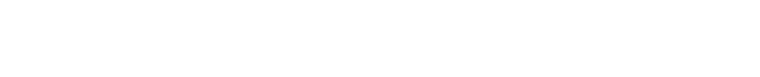




\section{An Econometric Analysis of Marketing Efficiency Determinants and Exports Determinants of Egyption Potatoes}

Ahmed Elfeel, Mahmoud Shafee, Nashwa Eltatawi, Heba Magraby

Agricultural marketing consists of all those business activities that go into getting a product such as potatoes in the hands of its ultimate consumers, The outputs of marketing are, consumers satisfaction with potatoes. The inputs are the various resources of labor' capital، and management that marketing firms use in the process, Efficient agricultural marketing، then can be defined as maximization of this input - output ratio. Thus، there is a necessity to develop an efficient potatoes marketing system to reduce the shortage in production.

Examine the objectives of this investigation include: (1) to analyze of the Egyptian potatoes production، consumption، and export capacity during the period 1998-2012, (2) To measure ' the potatoes marketing efficiency and its determinants, (3) To analyze the geographical distribution of potato exports and the determinants of its demand function. These objectives are of interest to the planners at various governmental and private marketing agencies to achieve their goals and to increase consumer satisfaction.
The main conclusion of the study indicate that: (1) Marketing efficiency for Egyptian potatoes ranges between a minimum at $18.1 \%$ in 2012 , and a maximum amounting to $60.0 \%$. The average for the study period amounts to $40.5 \%$ during the study period, (2) The marketing efficiency function showed a positive effect for the potatoes farm price, and the costs of production per ton .However, the retail price increase has a negative effect on potato marketing efficiency, (3) The geographical distribution of potato exports during the period 1998-2012 indicate, (a) That: Italy, Russia, Greece, Germany, and Lebanon took the first position in terms of imports of Egyptian potatoes by $78.8 \%$ of the average total quantity of Egyptian exports, (b) The Italian market is ranked first of these imports, (c) The main determinants of demand for Egyptian potato exports are: (i) The Egyptian local potatoes production capacity, (ii) The Egyptian potatoes exports price, (iii) The French potato export price, (4) The forecast of Egyptian potato exports shows that it amounts to 448.14 thousand tons in 2014 , to 460.2 thousand tons in 2015 , to 472.29 thousand tons in 2016 , and to 484.37 thousand tons in 2017. 


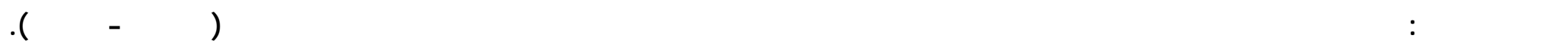

\begin{tabular}{|c|c|c|c|c|c|c|c|c|c|c|}
\hline كمية والردل & & & 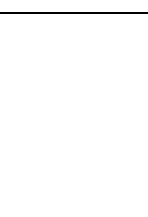 & & & & جمالم & & لجماليكمية & المتغيركة / \\
\hline 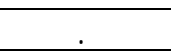 & r.E.rA & rદ१. $\cdot 1$ & $11.0 r$ & $r \mid . .1 \mu$ & 119.19 & 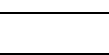 & $1 \% \wedge 7$ & $19 \wedge \varepsilon . \cdot 1 \mu$ & rYA.EV & 1991 \\
\hline $71 . \cdot \mu$ & rEr. $\cdot V$ & FIV.11 & $|\varepsilon .1|^{\mu}$ & YYA.OE & $1 \Lambda \cdot .14$ & $\Lambda^{\mu \mu}$ & 1171 & $1 \Lambda \cdot \Lambda . \wedge q \varepsilon$ & roo.ov & 1999 \\
\hline 91.40 & $170 . \varepsilon$. & 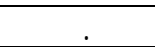 & $\Lambda . \Lambda \mathrm{V}$ & $177.1 \varepsilon$ & $18 \cdot .19$ & 1119 & 119. & IVาE.9| & $107.7 \mu$ & $r \ldots$ \\
\hline 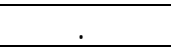 & $1 \wedge 9.01$ & 117.17 & $9 . \vee 0$ & IV7.7I & $17 \cdot . \mu \mathrm{V}$ & 1119 & $1 \mu \varepsilon \mu$ & $19 . \mu .1 \mu \varepsilon$ & $1 \wedge 0.0$ & $r \cdots 1$ \\
\hline $00 . \varepsilon 7$ & r ${ }^{\mu} \mu .90$ & $r r \cdot .70$ & 11.00 & 199.1 & $110 . v 9$ & $99 \mu$ & $1 \mu 7 \mid$ & 19^0.rIV & гч१.ґ^ & $r \cdots r$ \\
\hline $79.1 \%$ & $r \varepsilon \cdot . \cdot T$ & rYA.EO & IE.OH & $r \cdot \mu . \Lambda \Lambda$ & $|\varepsilon \Lambda . \varepsilon|$ & $1 \% \wedge 9$ & $\mid \mu \varepsilon \Lambda$ & $r \cdot \mu q . \mu O I$ & rq7.rq & $r \cdots \mu$ \\
\hline YH.YT & $\mu \cdots .1$ & rNO.Y. & IE. १V & rEદ.IV & $1 V 0.91$ & $10 . r$ & $10 \% 9$ & ro\&7.71. & rNI.KI & $r \cdot \varepsilon$ \\
\hline Vr.91 & rIY.rE & 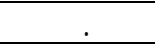 & IY.MY & $r \mid \cdot . \mu r$ & $19 V .0 \varepsilon$ & $11 \varepsilon 7$ & rYTo & 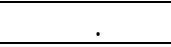 & $\mu q \cdot . \mu 0$ & $r \cdots 0$ \\
\hline $0 \Lambda . \cdot 1$ & 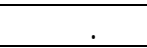 & ror. 1 & $10 . \Lambda r$ & rVV.7V & IVV. عo & IVYY & $1 \varepsilon 0$. & THוT.VqT & rา0.v9 & $r \cdots \tau$ \\
\hline 71.0 & 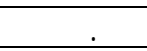 & $\varepsilon 70.0 \varepsilon$ & $1 \% .10$ & YYV. rV & זVา. हo & $19 \varepsilon$. & IVE⿱ & זVา.$\varepsilon า \varepsilon$ & rAT.ro & $r \cdots v$ \\
\hline$|r 7 . \varepsilon|$ & rAT.rq & EOr.E7 & $|\cdot . \varepsilon|$ & H"Y.rA & $\varepsilon \mu q . \varepsilon V$ & $r \cdot v$. & זrר7 & roTV. 0 . & PVI.19 & $r \cdots \Lambda$ \\
\hline$\varepsilon \mu .17$ & 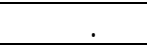 & $0 ૧ \varepsilon . \wedge \Lambda$ & $0 . \Lambda \Lambda$ & rq9. ${ }^{\mu \mu}$ & $7 \vee 7 . \varepsilon \varepsilon$ & rEस. & $r^{\mu} \cdot q$ & 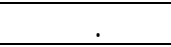 & $r 10.10$ & $r \ldots q$ \\
\hline VY.91 & $r 70.7$ & $\varepsilon \mid V . \mu^{\mu}$ & $\Lambda . r \varepsilon$ & $\mu \backslash \Lambda .0$ & $\varepsilon \mu\urcorner .{ }^{\mu} 0$ & rAT. & $r \mu .9$ & "广 & 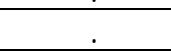 & $r .1$. \\
\hline IET.YE & ץEา.YV & $0 \cdot V .{ }^{\mu} I$ & $\mid \varepsilon .7 \varepsilon$ & PVI.10 & एवह.09 & $\varepsilon \cdot 7$. & rol. & हा ${ }^{\mu} \Lambda . \varepsilon \mu \mid$ & $7^{\mu} \varepsilon .9 V$ & $r .11$ \\
\hline vqщ. & r०१.१० & દ.૧.દV & T. & " $V^{\mu}$.EV & 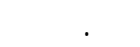 & or.. & r. & $\varepsilon \vee 0 \Lambda . \cdot \varepsilon \cdot$ & 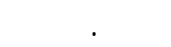 & $r \cdot i r$ \\
\hline
\end{tabular}

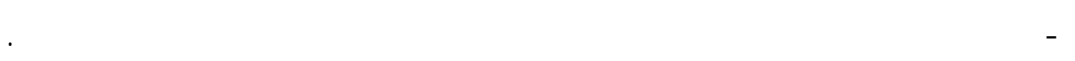

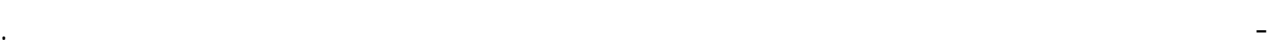

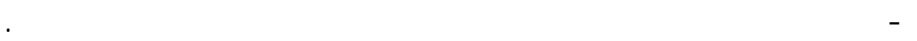

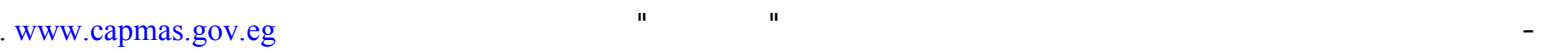

